\title{
Utilisation d'une cohomologie étale équivariante en topologie arithmétique
}

\author{
Baptiste Morin
}

\begin{abstract}
Sikora has given results which confirm the analogy between number fields and 3-manifolds. However, he has given proofs of his results which are very different in the arithmetic and in the topological case. In this paper, we show how to provide a unified approach to the results in the two cases. For this we introduce an equivariant cohomology which satisfies a localization theorem. In particular, we obtain a satisfactory explanation for the coincidences between Sikora's formulas which leads us to clarify and to extend the dictionary of arithmetic topology.
\end{abstract}

\section{Introduction}

Le calcul de la cohomologie étale du spectre $X$ de l'anneau d'entiers d'un corps de nombres $L$ laisse imaginer une analogie entre les corps de nombres et les variétés réelles de dimension trois. En effet, lorsque $L$ est totalement imaginaire, les groupes de cohomologie étale de $X$ à coefficients dans un faisceau abélien arbitraire sont nuls après la dimension trois (cf. [Den86, Proposition 4.6]). De plus, le théorème de dualité d'Artin-Verdier, analogue arithmétique de la dualité de Poincaré, fait à nouveau apparaître la dimension trois comme dimension maximale. Par ailleurs, le spectre d'un corps fini est, du point de vue de la topologie étale, un objet de dimension un dont le groupe fondamental est le complété profini de $\mathbb{Z}$. Pour cette raison, les points fermés de $X$ sont vus comme des noeuds dans une variété de dimension trois. Une extension galoisienne $L / K$ de groupe $G$ correspond à un revêtement galoisien $M \rightarrow M / G$ de variétés compactes de dimension trois. Dans cette situation, le groupe des classes $\mathrm{Cl}(L)$ et le quotient libre $U_{L} / \mu_{L}$ du groupe des unités de $L$ correspondent respectivement, en tant que modules galoisiens, à la partie de torsion $H_{\text {tor }}(M)$ et au quotient libre $H_{\text {free }}(M)$ du premier groupe d'homologie singulière de $M$ à coefficients entiers. Cette analogie est connue sous le nom de topologie arithmétique et nous renvoyons à [Rez99] pour un dictionnaire plus complet.

Lorsque le groupe de Galois est le groupe cyclique d'ordre premier $C_{p}$ et qu'il opère par automorphismes préservant l'orientation, le lieu de branchement du revêtement $M \rightarrow M / C_{p}$ est constitué d'un nombre fini de noeuds ramifiés, analogues topologiques des places finies ramifiées dans l'extension $L / K$. Cette situation est envisagée dans [Sik03]. A partir de la structure galoisienne des groupes $H_{\text {tor }}(M)$ et $H_{\text {free }}(M)$ (respectivement $\mathrm{Cl}(L)$ et $U_{L} / \mu_{L}$ ), Sikora y donne un encadrement du nombre $s$ de noeuds (respectivement de places finies) ramifiés. Il obtient des résultats en accord quasi-parfait avec le dictionnaire de la topologie arithmétique. Cependant, ses preuves sont basées sur des méthodes très différentes, puisqu'il utilise une cohomologie équivariante dans le contexte géométrique alors qu'il fait appel à à la théorie du corps de classes en arithmétique.

Received 12 February 2007, accepted in final form 21 May 2007, published online 23 January 2008.

2000 Mathematics Subject Classification 14F20, 11R04, 11R32.

Keywords: étale cohomology, localization theorem, arithmetic topology.

This journal is (c) Foundation Compositio Mathematica 2008. 
Le but de ce travail est de fournir des démonstrations analogues à ces résultats analogues afin de comprendre la coïncidence à priori très surprenante de ces résultats. Alors, ces résultats, leurs hypothèses et leurs démonstrations mettent en interaction la quasi-totalité des éléments du dictionnaire de la topologie arithmétique, et permettent ainsi de tester la validité de ces correspondances. Afin d'approfondir ce dictionnaire, la pertinence de certains de ses éléments et les contradictions offertes par d'autres sont alors mises en évidence.

La cohomologie équivariante modifiée utilisée dans [Sik03] pour traiter le cas des variétés topologiques satisfait essentiellement deux propriétés. Elle est d'une part l'aboutissement d'une suite spectrale dont le terme initial est de la forme $\widehat{H}^{p}\left(G ; H^{q}(M ; \mathbb{Z})\right) \Longrightarrow \widehat{H}_{G}^{p+q}(M ; \mathbb{Z})$. D'autre part, cette cohomologie équivariante satisfait un théorème de localisation, et par conséquent, ne 'voit' que le lieu de ramification. Dans la $\S 2$, nous définissons l'analogue en cohomologie étale de cette théorie et la suite spectrale $\widehat{H}^{p}\left(G ; H^{q}\left(X_{\text {et }} ; F\right)\right) \Longrightarrow \widehat{H}_{G}^{p+q}\left(X_{\text {et }} ; F\right)$ qui y aboutit. Les groupes $\widehat{H}_{G}^{q}\left(X_{\text {et }} ; F\right)$ ne sont définis que lorsque le groupe d'automorphismes $G$ est fini, et ne peuvent être intéressants que si les groupes de cohomologie de $F$ sont nuls à partir d'une certaine dimension $n$. Dans ce cas, les groupes $\widehat{H}_{G}^{q}\left(X_{\text {et }} ; F\right)$ s'identifient à partir de la dimension $n+1$ aux groupes de cohomologie mixte $H^{q}\left(X_{\text {et }} ; G ; F\right)$ (cf. la remarque 2.10), définis comme les invariants cohomologiques du topos des $G$-faisceaux d'ensembles sur $X_{\text {et }}$ (cf. [Gro66, 2]).

Nous démontrons dans la $\S 3$ le théorème de localisation énoncé ci-dessous.

THÉORÈme. Soit $X$ un schéma noethérien sur lequel un groupe fini $G$ opère fidèlement et de manière admissible. On note $Z$ le lieu de ramification du revêtement $X \rightarrow X / G$ et $\phi: \widetilde{Z} \rightarrow X$ la limite projective des voisinages étales de $Z$. Soit de plus $F$ un $G$-faisceau adapté (3.1) sur $X$. Alors le morphisme canonique $\widehat{H}_{G}^{*}\left(X_{\text {et }} ; F\right) \longrightarrow \widehat{H}_{G}^{*}\left(\widetilde{Z}_{\text {et }} ; \phi^{*} F\right)$ est un isomorphisme. Si de plus $F$ est de torsion et si $Z$ est contenu dans un ouvert affine, on a l'isomorphisme $\widehat{H}_{G}^{*}\left(X_{\mathrm{et}} ; F\right) \simeq \widehat{H}_{G}^{*}\left(Z_{\mathrm{et}} ; i^{*} F\right)$, où $i: Z \rightarrow X$ désigne l'immersion fermée canonique.

Ce théorème permet par exemple de calculer les groupes $\widehat{H}_{G}^{q}\left(X_{\text {et }} ; \mathbb{G}_{m}\right)$, lorsque $X$ est le spectre de l'anneau d'entiers d'un corps de nombres $L$. On trouve en particulier lorsque $G$ est abélien, l'isomorphisme $\widehat{H}_{G}^{0}\left(X_{\mathrm{et}} ; \mathbb{G}_{m}\right) \simeq \prod I_{\mathfrak{q}}$, où le produit est pris sur l'ensemble des places $\mathfrak{q}$ de $L^{G}$ et pour lesquelles $I_{\mathfrak{q}}$ désigne le sous-groupe d'inertie dans $G$. La suite spectrale établit donc un lien entre la ramification dans l'extension $L / L^{G}$ et la structure galoisienne des groupes $\mathrm{Cl}(L)$ et $U_{L}$. La première moitié de la $\S 4$ est consacrée à l'étude de cette suite spectrale et à certaines de ses conséquences. On obtient en particulier une démonstration des résultats de Sikora en théorie des nombres à l'aide de ces méthodes.

Nous exprimons dans la $\S 4.4$ la relation de dualité donnant les isomorphismes

$$
\widehat{H}_{G}^{n}\left(X_{\mathrm{et}} ; \mathbb{G}_{m}\right) \simeq \widehat{H}_{G}^{2-n}\left(X_{\mathrm{et}} ; \mathbb{Z}\right)^{D},
$$

d'ailleurs compatibles à la dualité induite par celle d'Artin-Verdier sur les termes initiaux des suites spectrales respectives. L'utilisation du $G$-faisceau $\mathbb{Z}$ permet alors de donner des preuves satisfaisantes du point de vue de la topologie arithmétique.

En effet, la preuve des résultats de [Sik03] de nature topologique et arithmétique respectivement s'articule comme suit. Il s'agit d'encadrer le nombre $s$ de noeuds (respectivement de places finies) ramifiés dans un revêtement $X \rightarrow X / C_{p}$ de variétés de dimension trois (respectivement de spectres d'anneaux d'entiers de corps de nombres) galoisien de groupe cyclique d'ordre premier $p$. Le théorème de localisation fournit les isomorphismes

$$
\widehat{H}_{C_{p}}^{n}(X ; \mathbb{Z}) \simeq \widehat{H}_{C_{p}}^{n}(Z ; \mathbb{Z}) \simeq \mathbb{F}_{p}^{s}
$$

où $Z$ désigne le lieu de ramification qui est constitué de $s$ noeuds (respectivement de $s$ places finies). 


\section{B. MORIN}

La suite spectrale

$$
\widehat{H}^{i}\left(C_{p} ; H^{j}(X ; \mathbb{Z})\right) \Longrightarrow \widehat{H}_{C_{p}}^{i+j}(X ; \mathbb{Z})
$$

donne ainsi des approximations successives du module $\mathbb{F}_{p}^{s}$ à partir des groupes $\widehat{H}^{i}\left(C_{p} ; H^{j}(X ; \mathbb{Z})\right)$. La dualité de Poincaré (respectivement d'Artin-Verdier) permet alors d'obtenir un encadrement du nombre $s$ en fonction de la dimension sur $\mathbb{F}_{p}$ des espaces $\widehat{H}^{0}\left(C_{p} ; H_{\text {tor }}(X)\right)$ et $\widehat{H}^{1}\left(C_{p} ; H_{\text {free }}(X)\right)$ (respectivement $\widehat{H}^{0}\left(C_{p} ; \mathrm{Cl}(L)\right)$ et $\left.\widehat{H}^{1}\left(C_{p} ; U_{L} / \mu\right)\right)$.

L'intérêt de ces groupes de cohomologie étale équivariante modifiée en topologie arithmétique est qu'ils sont les stricts analogues des mêmes groupes définis dans le contexte topologique, ce qui n'est pas le cas des premiers groupes de cohomologie équivariante non modifiée. Ceci vient renforcer l'analogie des preuves esquissées ci-dessus.

Cependant, l'utilisation de la topologie étale d'Artin-Verdier est nécessaire pour traiter le cas des extensions de corps de nombres non totalement imaginaires.

Pour terminer, nous tirons les conclusions de ce travail dans une cinquième section. Nous prenons alors clairement parti pour la première des deux versions (sensiblement différentes) du dictionnaire de la topologie arithmétique proposées par Reznikov dans [Rez99] et [Rez00]. On est ainsi amené à montrer comment la cohomologie de Lichtenbaum (conjecturale à ce jour; cf. [Fla06]), associée à la topologie Weil-étale, fournit naturellement un analogue arithmétique au groupe $H_{1}(M ; \mathbb{Z})$, dont le sous-groupe de torsion et le quotient libre maximal s'identifient, en tant que modules galoisiens, à $\mathrm{Cl}(L)$ et $U_{L} / \mu_{L}$ respectivement. Les calculs de Lichtenbaum confirment ainsi certaines correspondances de la topologie arithmétique. Cependant, il semble que l'analogue arithmétique du groupe fondamental $\pi_{1}(M)$ ne puisse être le groupe de Galois $G_{L}^{\mathrm{nr}}$ de l'extension maximale non ramifiée de $L$.

Ces considérations suggèrent que les résultats de [Sik03] sont deux manifestations d'un même phénomène, l'arithmétique apparaissant ici comme un 'cas particulier' du cadre topologique.

\section{Cohomologie étale équivariante modifiée}

On fixe un schéma $X$ sur lequel un groupe fini $G$ opère (à gauche) par automorphismes. Dans la suite, on appelle faisceau sur $X$ un faisceau de groupes abéliens pour la topologie étale et on note $\operatorname{Ab}(X)$ la catégorie des faisceaux sur $X$.

\subsection{La catégorie des $G$-faisceaux}

Nous rappelons quelques propriétés de cette catégorie indispensables pour la suite et pour lesquelles nous n'avons pas de référence.

DÉFinition 2.1. Soient $X$ un schéma muni d'une action d'un groupe fini $G$ et $F$ un faisceau sur $X$. On appelle $G$-linéarisation de $F$ la donnée d'une famille $\left(\varphi_{\sigma}\right)_{\sigma \in G}$ de morphismes de faisceaux $\varphi_{\sigma}: \sigma_{*} F \rightarrow F$, telle que les conditions suivantes soient satisfaites :

- $\varphi_{1}=\mathrm{Id}$;

- $\varphi_{\tau \sigma}=\varphi_{\tau} \circ \tau_{*}\left(\varphi_{\sigma}\right)$ (condition de cocycle).

Un $G$-faisceau est un faisceau muni d'une $G$-linéarisation. Un morphisme de $G$-faisceaux $\alpha$ : $F \rightarrow L$ est un morphisme de faisceaux $\alpha: F \rightarrow L$ tel que les diagrammes

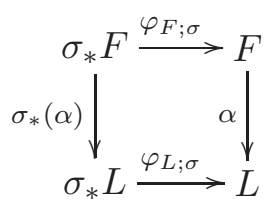


soient commutatifs, les structures de $G$-faisceaux sur $F$ et $L$ étant définies par les $G$-linéarisations $\left(\varphi_{F ; \sigma}\right)_{\sigma \in G}$ et $\left(\varphi_{L ; \sigma}\right)_{\sigma \in G}$. Les faisceaux usuels en topologie étale (par exemple $\mathbb{G}_{m}$ ou les faisceaux constants) sont naturellement munis de $G$-linéarisations (cf. [Gro57, 5.1]).

On note $\operatorname{Ab}(G ; X)$ la catégorie des $G$-faisceaux sur $X$ et de leurs morphismes.

Remarque 2.2. Etant donné deux $G$-faisceaux $F$ et $L$ sur $X$, on fait opérer $G$ sur le groupe $\operatorname{Hom}_{\mathrm{Ab}(X)}(F ; L)$ de la manière suivante. Si $\alpha: F \rightarrow L$ est un morphisme de faisceaux et $\sigma$ un élément de $G$, on pose $\sigma * \alpha:=\varphi_{L ; \sigma} \circ \sigma_{*}(\alpha) \circ \varphi_{F ; \sigma}^{-1}$. Alors $\operatorname{Hom}_{\mathrm{Ab}(G ; X)}(F ; L)$ est le sous-groupe des invariants de $\operatorname{Hom}_{\mathrm{Ab}(X)}(F ; L)$ sous l'action de $G$.

On montre alors facilement que la catégorie $\operatorname{Ab}(G ; X)$ est additive, puisque $\operatorname{Ab}(X)$ l'est. De plus, si $\alpha: F \rightarrow L$ est un morphisme de $G$-faisceaux, la $G$-linéarisation de $F$ en induit une sur $\operatorname{Ker}(\alpha)$, et celle définie sur $L$ en induit une sur $\operatorname{Coker}(\alpha)$. D'autre part, un isomorphisme de $G$-faisceaux est un morphisme de $G$-faisceaux qui est un isomorphisme en tant que morphisme de faisceaux. Donc si $\alpha$ est un morphisme dans $\operatorname{Ab}(G ; X)$, le morphisme $\bar{\alpha}: \operatorname{Coim}(\alpha) \rightarrow \operatorname{Im}(\alpha)$ est un isomorphisme de $G$-faisceaux. Autrement dit, $\operatorname{Ab}(G ; X)$ est une catégorie abélienne.

Remarque 2.3. Soit $\phi: X \rightarrow Y$ un morphisme de schémas sur lesquels le groupe $G$ opère. On dit que $X$ est muni d'une action de $G$ compatible à celle définie sur $Y$ lorsque $\phi$ commute à l'action de $G$. Dans ces conditions, $\phi_{*}$ est un foncteur de la catégorie des $G$-faisceaux sur $X$ dans celle des $G$-faisceaux sur $Y$. En effet, si $F$ est un $G$-faisceau sur $X$, la $G$-linéarisation de $F$ est transportée $\operatorname{sur} \phi_{*} F$ par le foncteur $\phi_{*}$. De même, $\phi^{*}$ est un foncteur exact de $\operatorname{Ab}(G ; Y)$ dans $\operatorname{Ab}(G ; X)$. De plus, si $F$ et $L$ sont des $G$-faisceaux sur $X$ et $Y$ respectivement, l'isomorphisme d'adjonction

$$
\operatorname{Hom}_{\mathrm{Ab}(X)}\left(\phi^{*} F ; L\right) \longrightarrow \operatorname{Hom}_{\mathrm{Ab}(X)}\left(F ; \phi_{*} L\right)
$$

commute à l'action de $G$ définie ci-dessus, car le morphisme d'ajonction $F \rightarrow \phi_{*} \phi^{*} F$ est un morphisme de $G$-faisceaux. En particulier, l'isomorphisme (1) identifie les sous-groupes des invariants $\operatorname{Hom}_{\mathrm{Ab}(G ; X)}\left(\phi^{*} F ; L\right)$ et $\operatorname{Hom}_{\mathrm{Ab}(G ; X)}\left(F ; \phi_{*} L\right)$. Ainsi, les foncteurs $\phi^{*}$ et $\phi_{*}$ entre les catégories de $G$-faisceaux sur $Y$ et $X$, sont adjoints. Il suit que $\phi_{*}$ préserve les $G$-faisceaux injectifs.

Proposition 2.4. La catégorie $\operatorname{Ab}(G ; X)$ possède suffisamment d'injectifs. De plus, un $G$-faisceau injectif est aussi injectif en tant que faisceau.

Démonstration. On vérifie facilement que le foncteur

$$
\begin{array}{rlc}
\operatorname{Sym}: \operatorname{Ab}(X) & \longrightarrow \operatorname{Ab}(G ; X) \\
L & \longmapsto \sum g_{*} L=\prod g_{*} L
\end{array}
$$

est adjoint à gauche du foncteur d'oubli $O u: \operatorname{Ab}(G ; X) \rightarrow \mathrm{Ab}(X)$. De plus, les foncteurs $g_{*}$ et $\sum$ sont exacts, donc Sym l'est aussi. On en déduit que le foncteur d'oubli préserve les injectifs.

D'autre part, Sym est aussi adjoint à droite du foncteur d'oubli. Si $F$ est un $G$-faisceau, on peut choisir un faisceau injectif $I$ dans lequel se plonge $F$. Ceci induit un morphisme injectif de $G$-faisceaux $F \rightarrow \operatorname{Sym}(I)$. De plus, l'isomorphisme d'adjonction

$$
\operatorname{Hom}_{\mathrm{Ab}(G ; X)}(-; \operatorname{Sym}(I)) \simeq \operatorname{Hom}_{\mathrm{Ab}(X)}(-; I) \circ O u
$$

montre que $\operatorname{Sym}(I)$ est un $G$-faisceau injectif. En effet, le foncteur d'oubli est exact et $I$ est injectif. Ainsi, tout $G$-faisceau se plonge dans un $G$-faisceau injectif.

Si $I^{*}(F)$ est une résolution injective de $F$ dans la catégorie $\operatorname{Ab}(G ; X)$, alors $I^{*}(F)(X)$ est un complexe de $\mathbb{Z}[G]$-modules. Il suit que les groupes de cohomologie usuels sont des $\mathbb{Z}[G]$-modules (à gauche).

Proposition 2.5. Si $\pi: X \rightarrow Y$ est un revêtement étale galoisien de groupe $G$, la catégorie des $G$-faisceaux sur $X$ est équivalente à celle des faisceaux sur $Y$. 


\section{B. MORIN}

Démonstration. Si $A$ est un faisceau sur $Y$, alors $\pi^{*} A$ est un $G$-faisceau sur $X$. En effet, $G$ opère trivialement sur $Y$ et $A$, et $\pi$ commute à l'action de $G$. D'autre part, si $F$ est un $G$-faisceau sur $X$, on définit un faisceau $\pi_{*}^{G} F$ sur $Y$ en posant, pour tout $Y$-schéma étale $U$,

$$
\pi_{*}^{G} F(U):=F\left(X \times_{Y} U\right)^{G} .
$$

Le morphisme $X \times_{Y} U \rightarrow U$ est un revêtement étale (non nécessairement connexe) galoisien de groupe $G$ quel que soit $U$ étale sur $Y$. Alors, si $A$ est un faisceau sur $Y$ et $U$ un $Y$-schéma étale, le groupe $A(U)$ s'identifie à $A\left(X \times_{Y} U\right)^{G}$ (cf. [Mil80, II.1.4]). On déduit facilement de ceci que les foncteurs $\pi^{*}$ et $\pi_{*}^{G}$ sont quasi-inverses l'un de l'autre.

\subsection{Cohomologie équivariante}

On fixe une résolution complète $W_{*}$ pour le groupe fini $G$ (cf. [Bro82, VI.3] ou [CE56, XII.3]). C'est un complexe de $\mathbb{Z}[G]$-modules (à gauche) libres de type fini et tel que la cohomologie du complexe $\operatorname{Hom}_{\mathbb{Z}[G]}\left(W_{*} ; M\right)$ donne les groupes de cohomologie modifiés $\widehat{H}^{*}(G ; M)$, pour tout $\mathbb{Z}[G]$-module $M$.

DÉfinition 2.6. Soient $F$ un $G$-faisceau et $0 \rightarrow F \rightarrow I^{0} \rightarrow I^{1} \rightarrow \cdots$ une résolution injective de $F$ dans $\operatorname{Ab}(G ; X)$. Alors $0 \rightarrow I^{0}(X) \rightarrow I^{1}(X) \rightarrow \cdots$ est un complexe de $\mathbb{Z}[G]$-modules. On note $\operatorname{Hom}_{\mathbb{Z}[G]}\left(W_{*} ; I^{*}(X)\right)$ le double complexe d'homomorphismes et

$$
\operatorname{Tot}^{n}\left(W_{*} ; I^{*}(X)\right):=\bigoplus_{i+j=n} \operatorname{Hom}_{\mathbb{Z}[G]}\left(W_{i} ; I^{j}(X)\right)
$$

le complexe (de cochaînes) total associé. La différentielle totale y est définie comme dans [Wei95, 2.7.4]. On définit les groupes de cohomologie étale équivariante modifiée de $X$ à coefficients dans $F$ de la manière suivante:

$$
\widehat{H}_{G}^{*}(X ; F):=H^{*}\left(\operatorname{Tot}^{*}\left(W_{*} ; I^{*}(X)\right)\right) .
$$

Ces groupes sont en fait définis comme l'aboutissement de la suite spectrale (la proposition 2.8). C'est ce qui justifie l'utilisation de la somme directe (et non du produit direct) dans la définition du complexe total. Cependant, il semble que cette définition ne donne des résultats intéressants que lorsqu'elle est appliquée à des $G$-faisceaux possédant une résolution flasque finie, c'est-à-dire quand la définition précédente du complexe total Hom prend du sens.

Proposition 2.7. La cohomologie du complexe $\operatorname{Tot}\left(W_{*} ; I^{*}(X)\right)$ ne dépend pas de la résolution injective de $F$ choisie.

Démonstration. Soient $I^{*}$ et $J^{*}$ deux résolutions injectives de $F$ dans $\operatorname{Ab}(G ; X)$. Il existe des morphismes de complexes $f: I^{*} \rightarrow J^{*}$ et $h: J^{*} \rightarrow I^{*}$ qui relèvent le morphisme Id $: F \rightarrow F$. Alors $h \circ f$ et $\operatorname{Id}_{\left(I^{*}\right)}$ relèvent l'identité et sont homotopes d'après [Wei95, 2.7.4]. De même, $f \circ h$ et $\operatorname{Id}_{\left(J^{*}\right)}$ sont homotopes. En appliquant à ceci le foncteur des sections globales, on obtient les morphismes de complexes de $\mathbb{Z}[G]$-modules $f_{X}: I^{*}(X) \rightarrow J^{*}(X)$ et $h_{X}: J^{*}(X) \rightarrow I^{*}(X)$ de sorte que $f_{X} \circ h_{X}$ et $h_{X} \circ f_{X}$ soient homotopes à l'identité du complexe de $\mathbb{Z}[G]$-modules correspondant. D'autre part, deux morphismes homotopes entre deux doubles complexes induisent les mêmes morphismes sur les groupes de cohomologie des complexes totaux associés (cf. [CE56, 15.6.1]). Les flèches $f_{X}$ et $h_{X}$ induisent donc des isomorphismes réciproques entre les groupes $H^{*}\left(\operatorname{Tot}\left(W_{*} ; I^{*}(X)\right)\right)$ et $H^{*}\left(\operatorname{Tot}\left(W_{*} ; J^{*}(X)\right)\right)$.

Proposition 2.8. Le groupe gradué $\widehat{H}_{G}^{*}(X ; F)$ est l'aboutissement d'une suite spectrale dont le terme initial est $E_{2}^{p ; q}(X ; F)=\widehat{H}^{p}\left(G ; H^{q}(X ; F)\right)$.

Démonstration. La première filtration du double complexe $\operatorname{Hom}\left(W_{*} ; I^{*}(X)\right)$ (que l'on note provisoirement $\left.D^{* *}\right)$ est régulière $(\mathrm{cf}$. $[\mathrm{CE} 56,15.6])$. La première suite spectrale de ce double complexe 
converge donc vers $\widehat{H}_{G}^{*}(X ; F):=H^{*}\left(\operatorname{Tot}\left(W_{*} ; I^{*}(X)\right)\right)$. Le terme initial de cette suite spectrale est $E_{2}^{p ; q}=H_{h}^{p}\left(H_{v}^{q}\left(D^{* *}\right)\right)$, où $H_{h}$ et $H_{v}$ désignent la cohomologie du double complexe $D^{* *}$ relativement aux différentielles horizontales et verticales. Le foncteur d'oubli $O u: \operatorname{Ab}(G ; X) \rightarrow \operatorname{Ab}(X)$ est exact et préserve les injectifs (cf. la proposition 2.4). L'égalité $E_{2}^{p ; q}=\widehat{H}^{p}\left(G ; H^{q}(X ; F)\right)$ en découle.

Cette suite spectrale ainsi que les groupes de cohomologie équivariante sont fonctoriels en $F$ et $X$. Plus précisément, un morphisme de $G$-faisceaux induit un morphisme entre les suites spectrales associées et un morphisme entre les groupes de cohomologie équivariante qui sont compatibles. Une suite exacte courte de $G$-faisceaux induit sur les groupes de cohomologie équivariante une suite exacte longue infinie dans les deux directions. D'autre part, soient $X$ et $Y$ deux schémas sur lesquels opère le groupe $G$ et $F$ un $G$-faisceau sur $Y$. Un morphisme $\pi: X \rightarrow Y$ compatible à l'action de $G$ induit un morphisme de la suite spectrale associée à $F$ sur $X$ dans celle associée à $\pi^{*} F$ sur $Y$, et de manière compatible, un morphisme sur les groupes de cohomologie équivariante.

La multiplication par le cardinal de $G$ annule toute la suite spectrale, y compris les groupes de cohomologie équivariante (cf. [Swa60, 1.2]). Si le groupe $G$ opère trivialement sur $X$ et $F$, la suite spectrale $E_{*}^{* ; *}(X ; F)$ est triviale, c'est à dire $E_{2}^{* ; *}(X ; F)=E_{\infty}^{* ; *}(X ; F)$ (cf. [Swa60, 1.2]).

Un faisceau sur $X$ est dit flasque si $\check{H}^{q}\left(\left\{U_{i} \rightarrow U\right\}_{i} ; F\right)=0$ pour tout $q \geqslant 1$ et tout recouvrement $\left\{U_{i} \rightarrow U\right\}_{i}$ (pour la topologie étale) d'un $X$-schéma étale $U$. D'après [Mil80, 3.2.12], $F$ est flasque si et seulement si $H^{q}(U ; F)=0$ pour tout $q \geqslant 1$ et tout $X$-schéma étale $U$. De tels faisceaux sont acycliques pour le foncteur des sections globales (cf. [Mil80, 3.1.8]). On appelle $G$-résolution acyclique de $F$ toute $G$-résolution de $F$ (i.e. toute résolution de $F$ dans $\operatorname{Ab}(G ; X))$ par des $G$ faisceaux acycliques pour le foncteur des sections globales.

Proposition 2.9. Toute G-résolution acyclique de $F$ permet de calculer les groupes de cohomologie étale équivariante de $X$ à coefficients dans $F$.

Démonstration. Soit $0 \rightarrow F \rightarrow I^{0} \rightarrow I^{1} \rightarrow \cdots$ une résolution injective de $F$ dans $\mathrm{Ab}(G ; X)$ et $0 \rightarrow F \rightarrow C^{0} \rightarrow C^{1} \rightarrow \cdots$ une $G$-résolution acyclique de $F$. On a un morphisme de complexes $f: C^{*} \rightarrow I^{*}$ qui relève l'identité d'ailleurs unique à homotopie près. En appliquant le foncteur des sections globales, on obtient le morphisme de complexe de $\mathbb{Z}[G]$-modules $f_{X}: C^{*}(X) \rightarrow I^{*}(X)$, qui donne des isomorphismes sur les groupes de cohomologie. Le morphisme de complexes $f_{X}$ induit un morphisme de doubles complexes $\operatorname{Hom}\left(W_{*} ; C^{*}(X)\right) \rightarrow \operatorname{Hom}\left(W_{*} ; I^{*}(X)\right)$, donc un morphisme au niveau des suites spectrales (convergentes) associées, qui est un isomorphisme dès la deuxième page. Ce qui précède définit un et un seul (car $f$ est défini à homotopie près) isomorphisme $H^{*}\left(\operatorname{Tot}\left(W_{*} ; C^{*}(X)\right)\right) \simeq H^{*}\left(\operatorname{Tot}\left(W_{*} ; I^{*}(X)\right)\right)(\operatorname{cf}$. [CE56, 15.3.2]).

Remarque 2.10. Soit $F$ un $G$-faisceau sur $X$ dont les groupes de cohomologie $H^{q}(X ; F)$ sont nuls pour $q \geqslant n+1$. Sous cette hypothèse, on construit dans la section suivante une $G$-résolution acyclique de $F$ de longueur $n$, qui d'après le théorème précédent, permet d'obtenir les groupes $\widehat{H}_{G}^{q}(X ; F)$. Soit $0 \rightarrow F \rightarrow C^{0} \rightarrow C^{1} \rightarrow \cdots \rightarrow C^{n} \rightarrow 0$ cette résolution.

La suite

$$
\cdots \rightarrow W_{i+1} \rightarrow W_{i} \rightarrow \cdots \rightarrow W_{0} \rightarrow \mathbb{Z} \rightarrow 0
$$

est une résolution de $\mathbb{Z}$ par des $\mathbb{Z}[G]$-modules projectifs. Les groupes de cohomologie du complexe total associé au double complexe $\left(\operatorname{Hom}_{\mathbb{Z}[G]}\left(W_{i} ; C^{j}(X)\right)\right)_{i, j \geqslant 0}$ (situé dans le premier quadrant) satisfont les conditions axiomatiques des foncteurs dérivés droits du foncteur (composé), qui à un $G$-faisceau $F$, associe le groupe $F(X)^{G}$ des sections globales invariantes sous l'action de $G$. Il s'agit donc des groupes de cohomologie mixte $H^{*}(X ; G ; F)$, définis comme les invariants cohomologiques du topos des $G$-faisceaux d'ensembles sur $X$ (cf. [Gro66, 2]).

On observe que les deux complexes totaux définissant respectivement les groupes $\widehat{H}_{G}^{*}(X ; F)$ et $H^{*}(X ; G ; F)$ coincident en dimension supérieure ou égale à $n$. Donc pour $q \geqslant n+1$, on a 


\section{B. MORIN}

l'identification

$$
\widehat{H}_{G}^{q}(X ; F) \simeq H^{q}(X ; G ; F) .
$$

\subsection{La résolution flasque de Godement}

Soit $F$ un faisceau sur $X$. La résolution de Godement est une résolution de $F$ par des faisceaux flasques. Lorsque $X$ est muni d'une action d'un groupe fini $G$ et que $F$ est un $G$-faisceau sur $X$, cette résolution doit être définie à partir d'un système de points géométriques stable sous l'action de $G$, afin de conserver une strucure équivariante. Il s'agit alors d'une $G$-résolution flasque qui permet, grâce à la proposition 2.9, de calculer la cohomologie étale équivariante de $X$. Nous verrons dans la $\S 3$ comment cette résolution peut être utilisée pour démontrer un théorème de localisation.

DÉfinition 2.11. On appelle $G$-système de points géométriques sur $X$ un ensemble $M$ de points géométriques $\alpha: \bar{\alpha} \rightarrow X$ satisfaisant les conditions suivantes.

- Si $x \in X$, il existe un point géométrique de $M$ dont l'image est $x$.

- Si $\alpha \in M$ et $g \in G$, alors $g(\alpha):=g \circ \alpha \in M$.

- $M$ est minimal pour ces propriétés.

On pose $X^{\prime}:=\prod_{\alpha \in M} \bar{\alpha}$. Les morphismes $(\alpha)_{\alpha \in M}$ induisent un morphisme $u: X^{\prime} \rightarrow X$ compatible à l'action de $G$ sur $X$ et $X^{\prime}$.

On construit un $G$-système de points géométriques de la manière suivante. Pour toute trajectoire $T$ de $G$ sur $X$, on choisit un point $x \in T$ et un point géométrique $\alpha_{T}$ dont l'image est $x$. On pose alors $X^{\prime}:=\coprod g\left(\alpha_{T}\right)$, où la somme est indexée sur l'ensemble des trajectoires $T$ et sur l'ensemble des éléments $g$ du groupe $G$.

On garde les notations de la définition précédente.

DÉfinition 2.12. On définit la résolution de Godement (équivariante)

$$
0 \rightarrow F \rightarrow C^{0}(F) \rightarrow C^{1}(F) \rightarrow \cdots
$$

de manière récurrente comme suit.

(i) On pose $C^{0}(F):=u_{*} u^{*}(F)$. On a un morphisme canonique injectif $\varepsilon: F \rightarrow C^{0}(F)$.

(ii) On pose $Z^{1}(F):=\operatorname{Coker}(\varepsilon)$ et $C^{1}(F):=C^{0}\left(Z^{1}(F)\right)$. Il y a un morphisme canonique $d^{0}$ : $C^{0}(F) \rightarrow C^{1}(F)$.

(iii) On définit par récurrence $Z^{n}(F):=\operatorname{Coker}\left(d^{n-2}\right)$ et $C^{n}(F):=C^{0}\left(Z^{n}(F)\right)$. Il y a un morphisme canonique $d^{n-1}: C^{n-1}(F) \rightarrow C^{n}(F)$.

Les faisceaux $C^{n}(F)$ sont flasques. De plus, $u$ est compatible à l'action de $G$, la catégorie $\operatorname{Ab}(G ; X)$ est abélienne et les foncteurs $u^{*}$ et $u_{*}$ sont adjoints. Il s'agit donc d'une $G$-résolution flasque.

Si $F$ est un $G$-faisceau sur $X$ et si $\varphi: U \rightarrow X$ est un morphisme étale, on a $C^{0}(F)(U)=$ $\prod_{\varphi \circ \beta \in M} F_{\beta}$, où le produit est pris sur l'ensemble des points géométriques $\beta$ de $U$ tels que $\varphi \circ \beta$ soit un élément de $M$.

Il sera utile dans la suite de disposer de $G$-résolutions flasques finies. Dans ce but, on définit pour tout entier $n$ la résolution de Godement de $F$ tronquée au cran $n$

$$
C_{(n)}^{*}(F): C^{0}(F) \rightarrow C^{1}(F) \rightarrow \cdots \rightarrow \operatorname{Im}\left(d^{n-1}: C^{n-1}(F) \rightarrow C^{n}(F)\right) \rightarrow 0 .
$$

Si pour tout $X$-schéma étale $U$ et pour tout $q \geqslant n+1$, on a $H^{q}(U ; F)=0$, alors $C_{(n)}^{*}(F)$ est une résolution flasque finie de $F$. En effet, les $C^{i}(F)$ sont flasques et on a

$$
H^{r}\left(U ; \operatorname{Im}\left(d^{n-1}\right)\right)=H^{n+r}(U ; F)=0
$$

pour tout $X$-schéma étale $U$ et pour tout $r \geqslant 1$. Le faisceau $\operatorname{Im}\left(d^{n-1}\right)$ est donc flasque. 


\section{Le théorème de localisation}

Nous démontrons un théorème permettant, sous certaines hypothèses, de calculer la cohomologie étale équivariante modifiée d'un schéma $X$ en se restreignant au sous-schéma fermé constitué des points de $X$ dont le groupe d'inertie est non trivial. Il s'agit de l'analogue d'un théorème de localisation pour les espaces topologiques de dimension finie (cf. [God58, V.12]) initialement démontré par Swan dans [Swa60]. Cette hypothèse de finitude se traduit ici par le fait que ce nouveau théorème de localisation ne s'applique qu'à des $G$-faisceaux 'adaptés' en un sens que nous allons définir.

La preuve de ce théorème comporte trois étapes. On commence par montrer que les groupes $\widehat{H}_{G}^{*}(X ; F)$ sont nuls, dès que le groupe $G$ opère sur $X$ sans inertie et que $F$ est adapté. Ceci permet ensuite de se concentrer aux $G$-voisinages étales des points ramifiés. On obtient alors le théorème en passant à la limite sur ces derniers.

\subsection{Le cas non ramifié}

Soit $F$ un faisceau sur $X$ possédant une résolution flasque finie, disons de longueur $n$. Alors les groupes $H^{q}(U ; F)$ sont nuls pour tout $X$-schéma étale $U$ et tout $q \geqslant n+1$. Réciproquement, on a remarqué dans la section précédente que cette condition permettait de construire une résolution flasque finie. Cette observation motive la définition suivante.

Définition 3.1. Soit $X \rightarrow Y$ un revêtement étale galoisien de groupe $G$ et $F$ un $G$-faisceau sur $X$. On dit que $F$ est adapte si il existe un entier $n$ de sorte que pour tout $Y$-schéma étale $V$ et pour tout $q \geqslant n+1$ on ait $H^{q}\left(V ; \pi_{*}^{G} F\right)=0$.

Cette condition de finitude sera nécessaire pour utiliser le lemme ci-dessous.

Lemme 3.2. Soit $M^{*}$ un complexe de cochaines de $\mathbb{Z}[G]$-modules tel que $M^{n}=0$ pour $n$ assez grand et pour $n$ négatif. On suppose de plus que $\widehat{H}^{i}\left(G ; M^{j}\right)=0$ pour tout $i$ et $j$. Alors on a $H^{*}\left(\operatorname{Tot}\left(W_{*} ; M^{*}\right)\right)=0$.

Démonstration. Dans ces conditions, la seconde filtration du double complexe $\operatorname{Hom}_{\mathbb{Z} G}\left(W_{*} ; M^{*}\right)$ est régulière ([CE56, II.15.6]), donc la seconde suite spectrale converge vers $H^{*}\left(\operatorname{Tot}\left(W_{*} ; M^{*}\right)\right)$. Le terme $E_{1}$ de cette suite spectrale est $\widehat{H}^{i}\left(G ; M^{j}\right)=0$, d'où $H^{*}\left(\operatorname{Tot}\left(W_{*} ; M^{*}\right)\right)=0$.

Proposition 3.3. Soit $\pi: X \rightarrow Y$ un revêtement étale galoisien de groupe $G$ avec $Y$ localement noethérien. Si $F$ est un $G$-faisceau adapté sur $X$, alors $\widehat{H}_{G}^{*}(X ; F)=0$.

Démonstration. On note abusivement $0 \rightarrow \pi_{*}^{G} F \rightarrow C^{0} \rightarrow C^{1} \rightarrow \cdots \rightarrow C^{n} \rightarrow 0$ la résolution de Godement de $\pi_{*}^{G} F$ tronquée au cran $n$. C'est une résolution flasque de $\pi_{*}^{G} F$. Comme $\pi^{*}$ est exact et qu'il préserve les faisceaux flasques, $0 \rightarrow \pi^{*} \pi_{*}^{G} F \rightarrow \pi^{*} C^{0} \rightarrow \pi^{*} C^{1} \rightarrow \cdots \rightarrow \pi^{*} C^{n} \rightarrow 0$ est une $G$-résolution flasque de $\pi^{*} \pi_{*}^{G} F=F$. On veut montrer que les $C^{j}(X)=\pi^{*} C^{j}(X)$ sont des $\mathbb{Z}[G]$-modules cohomologiquement triviaux.

Soit $S$ un sous groupe de $G$. Le morphisme $X \rightarrow X / S$ est un revêtement étale galoisien de groupe $S$ et $\phi: X / S \rightarrow Y$ est un morphisme étale (cf. [Gro71, V.3.3]). Les faisceaux $\phi^{*} C^{j}$ sont flasques et on a pour tout $q$ strictement positif (cf. [Mil80, III.2.6]) :

$$
H^{q}\left(S ; C^{j}(X)\right)=\check{H}^{q}\left(\{X \rightarrow X / S\} ; \phi^{*} C^{j}\right)=0,
$$

où l'on considère les groupes de cohomologie de Čech relatifs au recouvrement étale $\{X \rightarrow X / H\}$. Pour tout sous-groupe $S$ de $G$, les $C^{j}(X)$ sont donc $\Gamma^{S}$-acycliques. On en déduit que les $C^{j}(X)$ sont cohomologiquement triviaux (cf. [Ser68, IX, théorème 8]). En appliquant le lemme 3.2, on obtient $\widehat{H}_{G}^{*}(X ; F)=0$. 


\section{B. MORIN}

Remarque 3.4. Sous les hypothèses de la proposition précédente, la suite spectrale

$$
H^{p}\left(G ; H^{q}(X ; F)\right) \Rightarrow H^{p+q}(G ; X ; F)
$$

est celle d'Hochschild-Serre (cf. [Mil80, III.2.20]), et les groupes de cohomologie mixte $H^{*}(G ; X ; F)$ s'identifient aux $H^{*}\left(Y ; \pi_{*}^{G} F\right)$. Ces groupes sont en général non nuls. Afin de ne décrire que la ramification, et de permettre ainsi l'existence d'un théorème de localisation, les groupes $\widehat{H}_{G}^{*}(X ; F)$ doivent s'annuler dans cette situation, c'est à dire lorsque $X \rightarrow X / G=Y$ est un revêtement étale.

On dit qu'un groupe fini $G$ opère sur un schéma $X$ de façon admissible si $X$ est réunion d'ouverts affines invariants par $G$ ou encore si toute trajectoire de $G$ dans $X$ est contenue dans un ouvert affine. Le quotient $X / G$ existe à cette condition (cf. [Gro71, V.1.7]). Soit $X$ un schéma sur lequel un groupe fini $G$ opère de manière admissible et $\varphi: U \rightarrow X$ un morphisme étale. On suppose que $U$ est muni d'une action de $G$ compatible à celle définie sur $X$, c'est à dire que $\varphi$ commute à l'action de $G$. Si de plus le morphisme $\varphi$ est affine, l'action de $G$ sur $U$ est admissible. En effet, soit $\left(X_{i}\right)_{i}$ un recouvrement de $X$ par des ouverts affines de $X$ stables par $G$, alors $\left(\varphi^{-1}\left(X_{i}\right)\right)_{i}$ est un recouvrement de $U$ possédant les mêmes propriétés. De même, si $\varphi: U \rightarrow X$ est une immersion ouverte, l'action de $G$ sur $U$ est admissible. En effet, soit $T$ une trajectoire de $U$ et $W$ un ouvert affine de $X$ la contenant. On a $T \subset U \cap W \subset W$. Comme dans $W$, toute partie finie a un système fondamental de voisinages ouverts affines, il existe un voisinage ouvert affine de $T$ contenu dans $U \cap W$ donc dans $U$.

Si un groupe fini $G$ opère sur $X$ de manière admissible et sans inertie (i.e. tous les groupes d'inertie sont triviaux), alors $X \rightarrow X / G$ est un revêtement étale galoisien de groupe $G$ (cf. [Ray70, $\mathrm{X}$, corollaire 1] et [Gro71, V.1.8]). Si de plus $X$ est supposé localement noethérien, $X / G$ l'est aussi (cf. [Ray70, X, corollaire 2]).

Corollaire 3.5. Soit $X$ un schéma localement noethérien sur lequel un groupe $G$ opère de manière admissible et sans inertie. Soient de plus $F$ un $G$-faisceau adapté sur $X$ et $U$ un $X$-schéma étale muni d'une action de $G$ compatible à celle définie sur $X$. Si le morphisme $\varphi: U \rightarrow X$ est une immersion ouverte ou encore s'il est affine, alors $F \mid U$ est adapté et $\widehat{H}_{G}^{*}(U ; F \mid U)=0$.

Démonstration. On a le diagramme commutatif suivant.

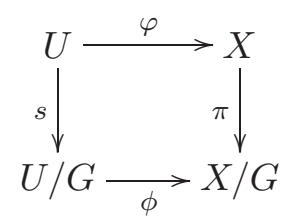

Soient $t \in U$ et $x=\varphi(t)$. On a $I_{t} \subseteq I_{x}=\{1\}$, où $I_{t}$ et $I_{x}$ sont les groupes d'inerties respectivement aux points $t$ et $x$. Le groupe $G$ opère sur $U$ sans inertie, donc $s$ est un revêtement étale galoisien. Les morphismes $s$ et $\phi \circ s=\pi \circ \varphi$ sont étales et $X / G$ est localement noethérien (car $X$ l'est). Il suit que $\phi$ est étale (cf. [Gro71, V.3.3]). Pour simplifier les notations, on pose $A:=\pi_{G}^{*} F$. On a

$$
F=\pi^{*} A \quad \text { et } \quad \varphi^{*} F=(\pi \circ \varphi)^{*} A=(\phi \circ s)^{*} A=s^{*}\left(\phi^{*} A\right) .
$$

Comme $\phi$ est étale, on a bien $H^{q}\left(V ; \phi^{*} A\right)=0$ pour tout $q \geqslant n$ et pour tout $(U / G)$-schéma étale $V$. Autrement dit, $\varphi^{*} F$ est adapté, car $s_{*}^{G}\left(\varphi^{*} F\right)=\phi^{*} A$. D'autre part, $\varphi$ est de type fini et $X$ est localement noethérien donc $U$ et $U / G$ le sont aussi. On obtient $\widehat{H}_{G}^{*}(U ; F \mid U)=0$ grâce à la proposition 3.3 .

Remarque 3.6. Lorsque $Y=X / G$ est localement noethérien et quasi-séparé, les résultats de cette section sont valables en remplaçant le cite étale de $Y$ par le cite étale restreint de $Y$ (cf. [GAV72, VII.3.2]). En effet, la catégorie des faisceaux sur ce cite est équivalente à celle des faisceaux sur 
le cite étale de $Y$ (qui est équivalente à celle des $G$-faisceaux sur $X$ ). De plus, tous les schémas envisagés dans cette section sont des objets du cite étale restreint de $Y$, c'est à dire des $Y$-schémas étales de présentation finie (cf. [Gro71, VIII.3.6]).

En effet, dans la preuve de (3.3) le morphisme $X \rightarrow X / G=Y$ est fini (cf. [Ray70, X, corollaire 1]). Il suit que $X / S \rightarrow Y$ est de type fini et séparé (cf. [Gro71, V.1.5]). Ces deux morphismes sont donc de présentation finie (quasi-séparés et de type fini). Dans la preuve de (3.5), le morphisme étale $U \rightarrow X$ est soit affine soit une immersion ouverte (et $X$ est localement noethérien), il est donc de présentation finie. De même, par (cf. [Gro71, VIII.3.6]), le morphisme $U / G \rightarrow Y$ est de présentation finie.

Soient $X \rightarrow Y$ un revêtement étale galoisien de groupe $G$ et $F$ un $G$-faisceau sur $X$. Lorsque $Y$ est localement noethérien et quasi-séparé, on dira parfois que $F$ est adapté sur $X$ s'il est adapté en tant que faisceau sur le cite étale restreint de $Y$. Alors $\widehat{H}_{G}^{*}(U ; F \mid U)=0$ pour tout morphisme équivariant $U \rightarrow X$ qui est soit étale et affine soit une immersion ouverte.

\subsection{Localisation à un voisinage étale des points ramifiés}

On suppose que $X$ est connexe et localement noethérien. Soit de plus un groupe fini $G$ opérant fidèlement sur $X$ et de manière admissible. On considère le morphisme $\pi: X \rightarrow X / G$. Soit $Z$ l'ensemble des points de $X$ dont les groupes d'inertie sont non triviaux. D'après [Ray70, X, corollaire 1], $Z$ est aussi l'ensemble des points en lesquels $\pi$ est ramifié. On en déduit que $Z$ est fermé dans $X$ (cf. [Gro71, I.3.3]). On note $X^{\prime}$ le complémentaire ouvert de $Z$ dans $X$. Le sous-schéma $X^{\prime}$ est localement noethérien, stable par $G$ et l'action de $G$ sur $X^{\prime}$ est admissible.

Un voisinage étale de $Z$ dans $X$ est un morphisme étale affine $\varphi: U \rightarrow X$ qui est un isomorphisme au-dessus de $Z$ (i.e. $U \times_{X} Z \rightarrow Z$ est un isomorphisme).

Définition 3.7. On appelle $G$-voisinage étale de $Z$ dans $X$ un voisinage étale $\varphi: U \rightarrow X$ muni d'une action de $G$ compatible à celle définie sur $X$, de sorte que $\varphi^{-1}(Z)$ soit d'intersection non vide avec chaque composante connexe de $U$.

Si $U$ peut être muni d'une action de $G$ qui fait de $\varphi: U \rightarrow X$ un $G$-voisinage étale de $Z$, alors cette action est définie de manière unique. En effet, soit $\sigma \in G$ et supposons qu'il existe $f, g: U \rightarrow U$ rendant le diagramme

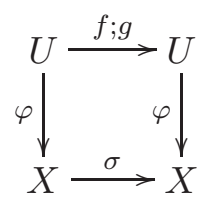

commutatif. Soient $p \in Z, q$ et $q^{\prime}$ les points de $U$ au-dessus de $p$ et $\sigma(p)$ respectivement et $V$ la composante connexe de $U$ contenant $q$. Alors $f(q)=g(q)=q^{\prime}$ et $g^{-1} \circ f(q)=q$. Comme $k(q) \simeq k(p), g^{-1} \circ f$ induit l'identité sur $k(q)$. On en déduit que $\left.g^{-1} \circ f\right|_{V}=\operatorname{Id}_{V}$ (cf. [Mil80, I.3.13]), puis que $g^{-1} \circ f$ induit l'identité sur chaque composante connexe de $U$.

ThÉorème 3.8. Soit $\varphi: U \rightarrow X$ un $G$-voisinage étale de $Z$ dans $X$ et $F$ un $G$-faisceau sur $X$. On suppose que $F \mid X^{\prime}$ est adapté. Alors il y a un isomorphisme canonique

$$
\widehat{H}_{G}^{*}(X ; F) \simeq \widehat{H}_{G}^{*}\left(U ; \varphi^{*} F\right) .
$$

Démonstration. On remarque d'abord que $\varphi: U \rightarrow X$ se factorise à travers $j: V \rightarrow X$, où $V:=\operatorname{Im}(\varphi)$ est un ouvert de $X$ (pour la topologie de Zariski) et $j$ l'inclusion. On note $\psi: U \rightarrow$ $V$ le morphisme satisfaisant $\varphi=j \circ \psi$. Alors $G$ opère sur $V, U$ et $X$ de manière compatible. 


\section{B. MORIN}

Autrement dit, le diagramme suivant est commutatif.

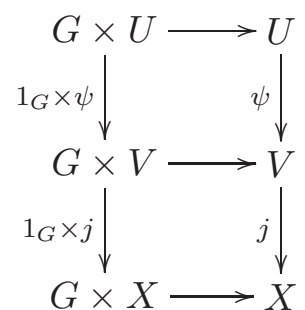

En particulier $j^{*} F$ et $\varphi^{*} F$ sont des $G$-faisceaux sur $V$ et $U$ respectivement. D'autre part, le morphisme $\varphi$ est affine donc $\psi$ et $j$ le sont aussi. On note $U^{\prime}:=X^{\prime} \times_{X} U, V^{\prime}:=X^{\prime} \times_{X} V$ et on commence par montrer le résultat suivant.

(a) Les groupes $\widehat{H}_{G}^{*}(X ; F)$ et $\widehat{H}_{G}^{*}\left(V ; j^{*} F\right)$ sont canoniquement isomorphes. En posant $Z^{n}=$ $Z^{n}(F)$, on a $C^{n}(F)(V)=C^{0}\left(Z^{n}\right)(V)=\prod_{j \circ \beta \in M}\left(Z^{n}\right)_{\beta}$. La résolution de Godement (non tronquée) donne la suite exacte de complexes de $\mathbb{Z}[G]$-modules

$$
C^{*}(F)(X) \rightarrow C^{*}(F)(V) \rightarrow 0 .
$$

Soit $K^{*}$ le complexe de $\mathbb{Z}[G]$-modules qui rend exacte la suite

$$
0 \rightarrow K^{*} \rightarrow C^{*}(F)(X) \rightarrow C^{*}(F)(V) \rightarrow 0 .
$$

Supposons avoir montré que $H^{*}\left(\operatorname{Tot}\left(W_{*} ; K^{*}\right)\right)=0$. Comme le foncteur $j^{*}$ est exact et qu'il préserve les faisceaux flasques,

$$
0 \rightarrow j^{*} F \rightarrow j^{*} C^{0}(F) \rightarrow \cdots \rightarrow j^{*} C^{n}(F) \rightarrow \cdots
$$

est une résolution de $j^{*} F$ par des faisceaux flasques. Le fait que $j: V \rightarrow X$ soit compatible à l'action de $G$ fait des $j^{*} C^{n}(F)$ des $G$-faisceaux et des $j^{*}\left(d^{n}\right)$ des morphismes de $G$-faisceaux. On a donc une $G$-résolution flasque de $j^{*}(F)$. D'autre part, $j^{*}\left(C^{n}(F)\right)(V)=C^{n}(F)(V)$, donc la cohomologie du complexe $C^{*}(F)(V)$ permet de calculer la cohomologie équivariante du faisceau $j^{*} F$. Les $W_{i}$ sont des $\mathbb{Z}[G]$-modules projectifs, donc la suite

$$
0 \rightarrow \operatorname{Tot}\left(W_{*} ; K^{*}\right) \rightarrow \operatorname{Tot}\left(W_{*} ; C^{*}(F)(X)\right) \rightarrow \operatorname{Tot}\left(W_{*} ; C^{*}(F)(V)\right) \rightarrow 0
$$

est encore exacte. La suite exacte longue de cohomologie qui lui est associée donne l'isomorphisme cherché.

Il suffit donc pour prouver (a) de montrer que $H^{*}\left(\operatorname{Tot}\left(W_{*} ; K^{*}\right)\right)=0$. Soient $N:=\{\alpha \in M$; $\operatorname{Im}(\alpha) \in V\}$ et $\Delta:=\{\alpha \in M ; \operatorname{Im}(\alpha) \in Z\}$. On a alors

$$
C^{n}(F)\left(X^{\prime}\right)=\prod_{\alpha \in M-\Delta}\left(Z^{n}\right)_{\alpha}, \quad C^{n}(F)\left(V^{\prime}\right)=\prod_{\alpha \in N-\Delta}\left(Z^{n}\right)_{\alpha},
$$

et

$$
K^{n}=\prod_{\alpha \in M-N}\left(Z^{n}\right)_{\alpha}=\prod_{\alpha \in(M-\Delta)-(N-\Delta)}\left(Z^{n}\right)_{\alpha} .
$$

On en tire la suite exacte

$$
0 \rightarrow K^{*} \rightarrow C^{*}(F)\left(X^{\prime}\right) \rightarrow C^{*}(F)\left(V^{\prime}\right) \rightarrow 0 .
$$

Les $W_{i}$ sont projectifs et la suite

$$
0 \rightarrow \operatorname{Tot}\left(W_{*} ; K^{*}\right) \rightarrow \operatorname{Tot}\left(W_{*} ; C^{*}(F)\left(X^{\prime}\right)\right) \rightarrow \operatorname{Tot}\left(W_{*} ; C^{*}(F)\left(V^{\prime}\right)\right) \rightarrow 0
$$

est exacte. La suite exacte longue de cohomologie associée devient donc

$$
\cdots \rightarrow H^{q}\left(\operatorname{Tot}\left(W_{*} ; K^{*}\right)\right) \rightarrow \widehat{H}_{G}^{q}\left(X^{\prime} ; F\right) \rightarrow \widehat{H}_{G}^{q}\left(V^{\prime} ; F\right) \rightarrow \cdots
$$


$X^{\prime}$ est localement nothérien et $G$ opère sur $X^{\prime}$ de façon admissible et sans inertie. De plus le morphisme $V^{\prime} \rightarrow X^{\prime}$ est une immersion ouverte compatible à l'action de $G$. Le corollaire 3.5 s'applique, d'où

et $H^{*}\left(\operatorname{Tot}\left(W_{*} ; K^{*}\right)\right)=0$.

$$
\widehat{H}_{G}^{q}\left(X^{\prime} ; F\right)=\widehat{H}_{G}^{q}\left(V^{\prime} ; F\right)=0,
$$

(b) Les groupes $\widehat{H}_{G}^{*}(V ; F)$ et $\widehat{H}_{G}^{*}\left(U ; \psi^{*} F\right)$ sont canoniquement isomorphes. Pour simplifier les notations, on désigne par $F$ le $G$-faisceau $j^{*} F$ sur $V$. De plus, $M$ est maintenant un $G$-système de points géométriques sur $V$, et $\Delta$ est toujours l'ensemble des points géométriques de $M$ dont l'image est un point de $Z$.

Le morphisme $\psi: U \rightarrow V$ est surjectif, donc pour tout $\alpha$ élément de $M$, il existe au moins un point géométrique de $U$ au-dessus de $\alpha$. En effet, soient $v$ l'image de $\alpha$ et $U_{v}=U \times_{V} \operatorname{Spec}(k(v))$ la fibre de $\psi$ au-dessus de $v$. Alors $U_{v}=\operatorname{Spec}(A)$ où $A$ est une $k(v)$-algèbre étale non nulle, car $\psi$ est étale et surjectif. Si l'on note $r$ le degré de $A$ sur $k(v)$, il existe exactement $r$ points géométriques de $U$ au-dessus de $\alpha$.

On a donc la suite exacte

$$
0 \rightarrow C^{*}(F)(V) \rightarrow C^{*}(F)(U) .
$$

En posant $I^{n}:=C^{n}(F)(U) / C^{n}(F)(V)$, on obtient la suite exacte

$$
0 \rightarrow C^{*}(F)(V) \rightarrow C^{*}(F)(U) \rightarrow I^{*} \rightarrow 0 .
$$

On suppose là aussi avoir montré que $H^{*}\left(\operatorname{Tot}\left(W_{*} ; I^{*}\right)\right)=0$. Comme ci-dessus,

$$
0 \rightarrow \psi^{*} F \rightarrow \psi^{*} C^{0}(F) \rightarrow \cdots \rightarrow \psi^{*} C^{n}(F) \rightarrow \cdots
$$

est une $G$-résolution flasque de $\psi^{*} F$, et le complexe $C^{*}(F)(U)$ permet de calculer la cohomologie équivariante du faisceau $\psi^{*} F$. On a de nouveau la suite exacte

$$
0 \rightarrow \operatorname{Tot}\left(W_{*} ; C^{*}(F)(V)\right) \rightarrow \operatorname{Tot}\left(W_{*} ; C^{*}(F)(U)\right) \rightarrow \operatorname{Tot}\left(W_{*} ; I^{*}\right) \rightarrow 0,
$$

et la suite exacte longue de cohomologie associée donne l'isomorphisme voulu.

Il reste à montrer que $H^{*}\left(\operatorname{Tot}\left(W_{*} ; I^{*}\right)\right)=0$. Si $\alpha \in \Delta, U$ possède un et un seul point géométrique au-dessus de $\alpha$, car $U$ est un voisinage étale de $Z$.

D'où

$$
C^{n}(F)(V)=\left(\prod_{\alpha \in \Delta} Z_{\alpha}^{n}\right) \times\left(\prod_{\alpha \in M-\Delta} Z_{\alpha}^{n}\right), \quad C^{n}(F)(U)=\left(\prod_{\alpha \in \Delta} Z_{\alpha}^{n}\right) \times\left(\prod_{\psi \circ \beta \in M-\Delta} Z_{\beta}^{n}\right)
$$

et

$$
I^{n}:=C^{n}(F)(U) / C^{n}(F)(V)=\prod_{\alpha \in M-\Delta}\left(\left(\prod_{\psi \circ \beta=\alpha} Z_{\beta}^{n}\right) / Z_{\alpha}^{n}\right)=C^{n}(F)\left(U^{\prime}\right) / C^{n}(F)\left(V^{\prime}\right),
$$

où $Z_{\alpha}^{n}$ se plonge diagonalement dans $\prod_{\psi \circ \beta=\alpha} Z_{\beta}^{n}$. On en déduit la suite exacte

$$
0 \rightarrow C^{*}(F)\left(V^{\prime}\right) \rightarrow C^{*}(F)\left(U^{\prime}\right) \rightarrow I^{*} \rightarrow 0,
$$

et enfin

$$
0 \rightarrow \operatorname{Tot}\left(W_{*} ; C^{*}(F)\left(V^{\prime}\right)\right) \rightarrow \operatorname{Tot}\left(W_{*} ; C^{*}(F)\left(U^{\prime}\right)\right) \rightarrow \operatorname{Tot}\left(W_{*} ; I^{*}\right) \rightarrow 0 .
$$

La suite exacte longue associée à cette dernière devient

$$
\cdots \rightarrow \widehat{H}_{G}^{q}\left(V^{\prime} ; F\right) \rightarrow \widehat{H}_{G}^{q}\left(U^{\prime} ; F\right) \rightarrow H^{q}\left(\operatorname{Tot}\left(W_{*} ; I^{*}\right)\right) \rightarrow \cdots .
$$

Le schéma $X^{\prime}$ est localement nothérien et $G$ opère sur $X^{\prime}$ de façon admissible et sans inertie. Le morphisme $V^{\prime} \rightarrow X^{\prime}$ est l'immersion d'un ouvert de $X^{\prime}$ stable sous l'action $G$ et le morphisme 


\section{B. MORIN}

$U^{\prime} \rightarrow X^{\prime}$ est affine (car $\varphi$ l'est), étale et compatible à l'action de $G$. Le corollaire 3.5 s'applique et montre que $\widehat{H}_{G}^{*}\left(V^{\prime} ; F\right)=\widehat{H}_{G}^{*}\left(U^{\prime} ; F\right)=0$. On obtient bien $H^{*}\left(\operatorname{Tot}\left(W_{*} ; I^{*}\right)\right)=0$, ce qui achève la preuve du théorème.

\subsection{Hensélisation}

On suppose que $X$ est un schéma connexe noethérien sur lequel le groupe fini $G$ opère fidèlement. On conserve les hypothèses et les notations de la $\S 2.2$. Soit $X$ un espace topologique muni d'une action d'un groupe fini $G$ et $Z$ un sous-espace fermé stable par $G$. Si $U$ est un ouvert de $X$ contenant $Z$, alors $\bigcap g(U)$ est un ouvert stable sous l'action de $G$ contenant $Z$ et contenu dans $U$. On procède de la même manière pour montrer le lemme suivant.

Lemme 3.9. L'ensemble des $G$-voisinages étales de $Z$ dans $X$ forme un système cofinal dans celui des voisinages étales de $Z$.

Démonstration. Soit $\varphi: U \rightarrow X$ un voisinage étale de $Z$ dans $X$. On note ${ }_{\sigma} U$ le $X$-schéma étale $\sigma \circ \varphi: U \rightarrow X$ et on pose $W:=\prod_{\sigma \in G}\left({ }_{\sigma} U\right)$, le produit fibré étant pris sur $X$. Le groupe $G$ opère sur lui-même par translations. Ceci définit une action de $G$ sur $W$ par permutation des coordonnées qui est compatible à l'action de $G$ sur $X$. Chacun des ${ }_{\sigma} U$ est un voisinage étale de $Z$ dans $X$. Il suit que $W \times{ }_{X} Z \rightarrow Z$ est un isomorphisme. D'autre part, $W \rightarrow X$ est affine puisque tous les ${ }_{\sigma} U \rightarrow X$ le sont. On note $G U$ le sous-schéma de $W$ formé des composantes connexes de $W$ contenant au moins un point s'envoyant dans $Z$. Alors $G U$ est un $G$-voisinage étale de $Z$ dans $X$ de sorte qu' il y a un morphisme canonique de $X$-schémas $G U \rightarrow U$. De plus, si $f: V \rightarrow U$ est un morphisme de $X$-schémas, $f$ induit canoniquement un morphisme $G V \rightarrow G U$ au-dessus de $X$ et compatible à l'action de $G$.

On note $\widetilde{Z}$ la limite projective des voisinages étales de $Z$ dans $X$. D'après ce qui précède, c'est aussi la limite projective des $G$-voisinages étales de $Z$ dans $X$. L'action de $G$ sur ces derniers passe à la limite pour donner une action de $G$ sur $\widetilde{Z}$ compatible à celle définie sur $X$. D'autre part, lorsque $X$ est noethérien, tout voisinage étale $U$ de $Z$ dans $X$ est noethérien (en particulier quasi-compact) puisque le morphisme $U \rightarrow X$ est de type fini.

On note $i: Z \rightarrow X, s: Z \rightarrow \widetilde{Z}$ et $\phi: \widetilde{Z} \rightarrow X$ les morphismes canoniques. Ces morphismes commutent à l'action de $G$ et satisfont $i=\phi \circ s$.

ThÉorème 3.10. Soit $F$ un $G$-faisceau sur $X$ tel que $F \mid X^{\prime}$ soit adapté. Alors le morphisme canonique $\widehat{H}_{G}^{*}(X ; F) \rightarrow \widehat{H}_{G}^{*}\left(\widetilde{Z} ; \phi^{*} F\right)$ est un isomorphisme.

Démonstration. On note $0 \rightarrow F \rightarrow C^{0} \rightarrow \cdots$ la résolution de Godement de $F$. On a les égalités suivantes (où toutes les limites inductives sont prises sur l'ensemble des $G$-voisinages étales de $Z$ dans $X)$ :

$$
\begin{aligned}
& \widehat{H}_{G}^{*}(X ; F)=\underline{\lim } \widehat{H}_{G}^{*}(U ; F) \\
& =H^{*}\left(\underset{\lim }{\longrightarrow} \operatorname{Tot}^{*}\left(W_{*} ; C^{*}(U)\right)\right) \\
& =H^{*}\left(\bigoplus_{i+j=*} \lim _{\longrightarrow} \operatorname{Hom}_{\mathbb{Z}[G]}\left(W_{i} ; C^{j}(U)\right)\right) \\
& =H^{*}\left(\bigoplus_{i+j=*} \operatorname{Hom}_{\mathbb{Z}[G]}\left(W_{i} ; \lim _{\longrightarrow} C^{j}(U)\right)\right) \\
& =H^{*}\left(\bigoplus_{i+j=*} \operatorname{Hom}_{\mathbb{Z}[G]}\left(W_{i} ; \phi^{*} C^{j}(F)(\widetilde{Z})\right)\right) \\
& =\widehat{H}_{G}^{*}\left(\widetilde{Z} ; \phi^{*} F\right) \text {. }
\end{aligned}
$$


L'égalité (2) est vraie car tous les morphismes $\widehat{H}_{G}^{*}(U ; F) \rightarrow \widehat{H}_{G}^{*}(V ; F)$ sont des isomorphismes. Etant donné un système inductif de complexes de $\mathbb{Z}[G]$-modules, la limite inductive commute avec la cohomologie, ce qui donne (3). L'égalité (4) est vraie car les limites inductives commutent avec les sommes directes. L'égalité (5) est vraie car les $W_{i}$ sont des $\mathbb{Z}[G]$-modules libres de type fini. Le foncteur $\phi^{*}$ est exact, donc $0 \rightarrow \phi^{*} F \rightarrow \phi^{*} C^{0} \rightarrow \cdots$ est une résolution de $\phi^{*} F$. Les voisinages étales sont quasi-compacts et les morphismes de transition sont affines, donc les morphismes $\lim H^{q}\left(U ; C^{j}\right) \rightarrow H^{q}\left(\widetilde{Z} ; \phi^{*} C^{j}\right)$ sont bijectifs. Ainsi, les faisceaux $\phi^{*} C^{j}$ sont acycliques pour le foncteur des sections globales. Les égalités (6) et (7) s'en déduisent.

Supposons que $Z$ soit contenu dans un ouvert affine $\operatorname{Spec}(A)$. Le sous-schéma fermé $Z$ est alors défini par un idéal $I=\sqrt{I}$ de $A$ de sorte que $Z=V(I)$ et $Z \simeq \operatorname{Spec}(A / I)$. Si $(\widetilde{A} ; \widetilde{I})$ désigne l'hensélisé du couple $(A ; I)$ (cf. [Ray70, XI]), alors $\widetilde{Z}:=\operatorname{Spec}(\widetilde{A})$.

Corollaire 3.11. On conserve les hypothèses du théorème 3.10. Lorsque $F$ est de torsion et que $Z$ est contenu dans un ouvert affine, le morphisme canonique

$$
\widehat{H}_{G}^{*}(X ; F) \rightarrow \widehat{H}_{G}^{*}\left(Z ; i^{*} F\right)
$$

est un isomorphisme.

Démonstration. Soit $s: Z \rightarrow \widetilde{Z}$ l'immersion fermé canonique. Comme $Z$ est contenu dans un ouvert affine, $\widetilde{Z}$ est affine. De plus, le $G$-faisceau $\phi^{*} F$ sur $\widetilde{Z}$ est de torsion lorsque $F$ l'est. Soient $I^{*}$ et $J^{*}$ des $G$-résolutions injectives de $\phi^{*} F$ et $s^{*} \phi^{*} F=i^{*} F$ respectivement. Le morphisme canonique de complexes de $\mathbb{Z}[G]$-modules $I^{*}(\widetilde{Z}) \rightarrow J^{*}(Z)$ induit un morphisme de suites spectrales et des isomorphismes sur les groupes de cohomologie étale (cf. [Hub93, 0.1]). Comme les suites spectrales en question convergent respectivement vers $\widehat{H}_{G}^{*}(\widetilde{Z} ; F)$ et $\widehat{H}_{G}^{*}\left(Z ; s^{*} F\right)$, ces deux groupes (gradués) sont isomorphes. En utilisant le théorème 3.10, on obtient le résultat annoncé.

Remarque 3.12. Soit $F$ un $G$-faisceau quelconque sur $X$ qui est adapté sur $X^{\prime}$. Pour appliquer la preuve précédente à $F$, il suffit de montrer que les morphismes canoniques $H^{q}\left(\widetilde{Z} ; \phi^{*} F\right) \rightarrow H^{q}\left(Z ; i^{*} F\right)$ sont bijectifs. Cette condition est toujours vérifiée lorsque $\widetilde{Z}$ est une somme finie de spectres d'anneaux locaux henséliens (cf. [GAV72, VIII 8.6]).

Soit $F$ un faisceau de torsion sur un schéma $X$. Pour tout nombre premier $l$, on note $F(l)$ le sous-faisceau de $l$-torsion de $F$. Le morphisme canonique $\bigoplus_{l} F(l) \rightarrow F$ est un isomorphisme.

Proposition 3.13. Soit $G$ un groupe fini opérant fidèlement et de manière admissible sur $X$. Si $X / G$ est un ouvert du spectre de l'anneau d'entiers d'un corps de nombres totalement imaginaire, alors tout $G$-faisceau sur $X$ est adapté sur $X^{\prime}$.

Soit $X$ de type fini et séparé $\operatorname{sur} \operatorname{Spec}(\mathbb{Z})$, et soit $F$ un $G$-faisceau de torsion sur $X$. Si $F(2)=0$ ou si aucun corps résiduel de $X$ n'est ordonnable, alors $F$ est adapté sur $X^{\prime}$.

Soit $X$ un schéma de type fini séparé sur un corps $k$, sur lequel $G$ opère par $k$-automorphismes. Si $k$ est de $l$-dimension cohomologique finie, tout $G$-faisceau de l-torsion sur $X$ est adapté sur $X^{\prime}$. Si $k$ est de dimension cohomologique finie, tout $G$-faisceau de torsion sur $X$ est adapté sur $X^{\prime}$.

Démonstration. Si $X / G$ est un ouvert du spectre de l'anneau d'entiers d'un corps de nombres totalement imaginaire, tout $X^{\prime} / G$-schéma étale (connexe) est aussi un ouvert du spectre de l'anneau d'entiers d'un corps de nombres totalement imaginaire. Dans ce cas, le résultat est une conséquence de $[$ Den86, 4.6].

On note $S$ la base $\operatorname{Spec}(\mathbb{Z})$ ou $\operatorname{Spec}(k)$. Soient $X$ de type fini et séparé sur $S$, et $F$ comme dans l'énoncé. L'immersion ouverte $X^{\prime} \rightarrow X$ est séparée et de type fini, car $X$ est noethérien. Ainsi $X^{\prime}$ est séparé et de type fini sur $S$. D'après [Gro71, V.1.5], le schéma $X^{\prime} / G$ est aussi de type fini et séparé sur $S$. 


\section{B. MORIN}

On pose $\pi: X^{\prime} \rightarrow X^{\prime} / G$ et $A:=\pi_{*}^{G} F$. Le faisceau $A$ est de torsion (respectivement de $l$-torsion) si $F$ l'est. D'après la remarque (3.6), il suffit de montrer qu'il existe un entier $n$ de sorte que $H^{q}(U ; A)=0$ pour tout $q \geqslant n+1$ et pour tout $U$ étale de présentation finie sur $X^{\prime} / G$. Dans ce cas, le schéma $U$ est quasi-compact et quasi-séparé, donc la cohomologie étale de $U$ commute aux limites inductives filtrantes de faisceaux (cf. [GAV72, VII.3.2]).

On pose $n:=2 \operatorname{dim}(X)+1 \operatorname{pour} S=\operatorname{Spec}(\mathbb{Z})$ et $n:=2 \operatorname{dim}(X)+\operatorname{cd}_{l}(k)$ (respectivement $n:=2 \operatorname{dim}(X)+\operatorname{cd}(k))$ pour $S=\operatorname{Spec}(k)$. On obtient, pour tout $q \geqslant n+1$,

$$
H^{q}(U ; A)=\bigoplus_{l} H^{q}(U ; A(l))=0 .
$$

En effet, le $S$-schéma $U$ est de type fini, donc la deuxième égalité est vraie grâce à [GAV72, X.6.2] et [Mil80, VI.1.4]. Ainsi, le $G$-faisceau $F$ est adapté sur $X^{\prime}$.

\section{Application à la topologie arithmétique}

Soit $X$ le spectre de l'anneau d'entiers $D$ d'un corps de nombres $L$ sur lequel un groupe fini $G$ opère. On pose $K:=L^{G}, Y=X / G=\operatorname{Spec}\left(D^{G}\right)$ et on considère l'extension galoisienne $L / K$. On suppose $L$ et $K$ totalement imaginaires. Les groupes de cohomologie étale du faisceau du groupe multiplicatif sur le site $X_{\text {et }}$ sont donnés ci-dessous (cf. [Maz73]).

$$
H^{q}\left(X ; \mathbb{G}_{m}\right)= \begin{cases}U_{L} & \text { pour } q=0, \\ \mathrm{Cl}(L) & \text { pour } q=1, \\ 0 & \text { pour } q=2, \\ \mathbb{Q} / \mathbb{Z} & \text { pour } q=3, \\ 0 & \text { pour } q \geqslant 4,\end{cases}
$$

où $U_{L}$ désigne le groupe des unités de $L, \mu$ le groupe cyclique des racines de l'unité et $\mathrm{Cl}(L)$ le groupe des classes. L'opération de $G$ sur les groupes $U_{L}$ et $\mathrm{Cl}(L)$ (en tant que groupes de cohomologie du $G$ faisceau $\left.\mathbb{G}_{m}\right)$ est l'action naturelle. De plus, le groupe de Galois opère trivialement sur $H^{3}\left(X ; \mathbb{G}_{m}\right)=$ $\mathbb{Q} / \mathbb{Z}$.

Le morphisme canonique $\pi: X \rightarrow Y$ est fini. On note $Z$ le sous-schéma fermé de $X$ constitué des points en lesquels $\pi$ est ramifié, $X^{\prime}$ son complémentaire ouvert et $s$ le cardinal de $Z$.

\subsection{Calcul des groupes $\widehat{H}_{G}^{*}\left(X ; \mathbb{G}_{m}\right)$}

Le quotient $X^{\prime} / G$ est un ouvert de $Y$. Soit $V$ un $X^{\prime} / G$-schéma étale. Alors $V$ est un ouvert du spectre de l'anneau d'entiers d'un corps de nombres totalement imaginaire et on a $H^{q}\left(V ; \mathbb{G}_{m}\right)=0$ pour tout $q \geqslant 4$ (cf. [Mil96, 2.2.1]). Le faisceau $\mathbb{G}_{m} \mid X^{\prime}$ est adapté donc le théorème de localisation s'applique dans ce cas.

Soient $\mathfrak{a}$ le produit des idéaux $\left(\mathfrak{p}_{i}\right)_{1 \leqslant i \leqslant s}$ de $D$ ramifiés dans l'extension $L / K$ et $Z=V(\mathfrak{a})$. Soient $(\widetilde{D} ; \widetilde{\mathfrak{a}})$ l'hensélisé du couple $(D ; \mathfrak{a})$ et $\widetilde{Z}:=\operatorname{Spec}(\widetilde{D})$ (cf. [Ray70]). Alors $\widetilde{D}=\prod_{1 \leqslant i \leqslant s} \widetilde{D}_{\mathfrak{p}_{i}}$, où $\widetilde{D}_{\mathfrak{p}_{i}}$ est l'anneau local constitué des éléments de la complétion de $D$ pour la valeur absolue $\mathfrak{p}_{i}$-adique qui sont algébriques sur $\mathbb{Q}$. C'est un anneau de valuation discrète hensélien à corps résiduel fini. On note $U_{\mathfrak{p}_{i}}$ le groupe des unités de $\widetilde{D}_{\mathfrak{p}_{i}}$ et $\widetilde{Z}_{i}$ le spectre de $\widetilde{D}_{\mathfrak{p}_{i}}$.

Le théorème de localisation donne l'isomorphisme $\widehat{H}_{G}^{*}\left(X ; \mathbb{G}_{m}\right) \simeq \widehat{H}_{G}^{*}\left(\widetilde{Z} ; \mathbb{G}_{m}\right)$. D'autre part, on a $\widetilde{Z}=\amalg \widetilde{Z}_{i}$ et $H^{q}\left(\widetilde{Z} ; \mathbb{G}_{m}\right)=\prod H^{q}\left(\widetilde{Z}_{i} ; \mathbb{G}_{m}\right)=\prod U_{\mathfrak{p}_{i}}$ pour $q=0$ et 0 sinon. En effet, $H^{q}\left(\widetilde{Z}_{i} ; \mathbb{G}_{m}\right) \simeq$ $H^{q}\left(\operatorname{Spec}\left(D / \mathfrak{p}_{i}\right) ; \mathbb{G}_{m}\right)$ pour tout $q \geqslant 1$ (cf. [Str84, 4.1]), or ces groupes sont nuls car un corps fini est $\mathrm{C} 1$ et donc de dimension cohomologique tout au plus un (cf. [Ser65]). La suite spectrale de 
UtiLisATION D’Une COHOMOLOGIE ÉTALE ÉQUIVARIANTE EN TOPOLOGIE ARITHMÉTIQUE

cohomologie équivariante de $\mathbb{G}_{m}$ sur $\widetilde{Z}$ dégénère et on obtient

$$
\widehat{H}_{G}^{*}\left(X ; \mathbb{G}_{m}\right) \simeq \widehat{H}_{G}^{*}\left(\widetilde{Z} ; \mathbb{G}_{m}\right)=\widehat{H}^{*}\left(G ; \prod_{1 \leqslant i \leqslant s} U_{\mathfrak{p}_{i}}\right) .
$$

Si $\mathfrak{q}$ est un idéal premier de $K$, on pose $U^{\mathfrak{q}}:=\prod_{\mathfrak{p} \mid \mathfrak{q}} U_{\mathfrak{p}}$ et on a la décomposition en $\mathbb{Z}[G]$ modules homogènes $\prod_{1 \leqslant i \leqslant s} U_{\mathfrak{p}_{i}}=\prod_{\mathfrak{q} \in \Omega} U^{\mathfrak{q}}$, où $\Omega$ désigne l'ensemble des idéaux premiers de $K$ qui se ramifient dans $L$. Lorsque $\mathfrak{p}$ est un idéal premier de $L$, on note $G_{\mathfrak{p}}$ le groupe de décomposition en l'idéal $\mathfrak{p}$. Alors $U^{\mathfrak{q}}$ est le $\mathbb{Z}[G]$-module induit $M_{G}^{G_{\mathfrak{p}}}\left(U_{\mathfrak{p}}\right)$, où l'on a choisi un idéal p premier de $L$ divisant q. Finalement, on a l'identification

$$
\widehat{H}^{p}\left(G ; \prod_{1 \leqslant i \leqslant s} U_{\mathfrak{p}_{i}}\right)=\prod_{\mathfrak{q} \in \Omega} \widehat{H}^{p}\left(G_{\mathfrak{p}} ; U_{\mathfrak{p}}\right),
$$

où l'on a choisi, pour tout $\mathfrak{q}$ dans $\Omega$, un idéal $\mathfrak{p}$ premier de $L$ divisant $\mathfrak{q}$.

Les relations (8) et (9) montrent le théorème suivant.

ThÉORÈme 4.1. On a l'isomorphisme

$$
\widehat{H}_{G}^{n}\left(X ; \mathbb{G}_{m}\right) \simeq \prod \widehat{H}^{n}\left(G_{\mathfrak{p}} ; U_{\mathfrak{p}}\right),
$$

où le produit est pris sur tous les idéaux premiers non nuls de $K$.

La théorie du corps de classe local (cf. [Mil96, I, Appendix A]) montre que le groupe $\widehat{H}^{0}\left(G_{\mathfrak{p}} ; U_{\mathfrak{p}}\right)$ est isomorphe au sous-groupe d'inertie $I_{\mathfrak{q}}^{(a b)}$ de l'abélianisé du groupe de décomposition $G_{\mathfrak{p}}$, c'est à dire le noyau du morphisme $G_{\mathfrak{p}}^{\text {ab }} \rightarrow \operatorname{Gal}(k(\mathfrak{p}) / k(\mathfrak{q}))$.

Soit $B$ l'anneau d'entiers de $K$ et $\widetilde{B}_{\mathfrak{q}}$ l'anneau de valuation discrète hensélien obtenu comme ci-dessus. On note $U_{\mathfrak{q}}$ le groupe des unités de $\widetilde{B}_{\mathfrak{q}}, L_{\mathfrak{p}}$ et $K_{\mathfrak{q}}$ les corps de fractions de $\widetilde{D}_{\mathfrak{p}}$ et $\widetilde{B}_{\mathfrak{q}}$. On considère la suite exacte de $\mathbb{Z}\left[G_{\mathfrak{p}}\right]$-modules

$$
0 \rightarrow U_{\mathfrak{p}} \rightarrow L_{\mathfrak{p}}^{\times} \rightarrow \mathbb{Z} \rightarrow 0
$$

où la flèche $L_{\mathfrak{p}}^{\times} \rightarrow \mathbb{Z}$ est donnée par la valuation $\mathfrak{p}$-adique. En utilisant le fait que l'extension $L_{\mathfrak{p}} / K_{\mathfrak{q}}$ est galoisienne et le théorème de Hilbert 90, la suite exacte longue de cohomologie donne la suite exacte

$$
0 \rightarrow U_{\mathfrak{q}} \rightarrow K_{\mathfrak{q}}^{\times} \rightarrow \mathbb{Z} \rightarrow \widehat{H}^{1}\left(G_{\mathfrak{p}} ; U_{\mathfrak{p}}\right) \rightarrow 0 .
$$

La restriction de la valuation $\mathfrak{p}$-adique à $K_{\mathfrak{q}}^{\times}$a pour image $e_{\mathfrak{q}} \mathbb{Z}$, où $e_{\mathfrak{q}}$ désigne l'indice de ramification associé à $\mathfrak{q}(L / K$ est galoisienne, donc cet entier ne dépend que de $\mathfrak{q})$. La dernière suite exacte permet donc d'identifier les groupes $\widehat{H}^{1}\left(G_{\mathfrak{p}} ; U_{\mathfrak{p}}\right)$ et $\mathbb{Z} / e_{\mathfrak{q}} \mathbb{Z}$. Le corollaire suivant résume ces résultats.

Corollaire 4.2. On a les identifications suivantes, où les trois produits sont pris sur tous les idéaux premiers (non nuls) de $K$ :

- $\widehat{H}_{G}^{0}\left(X ; \mathbb{G}_{m}\right)=\prod I_{\mathfrak{q}}^{(a b)} ;$

- $\widehat{H}_{G}^{1}\left(X ; \mathbb{G}_{m}\right)=\prod \mathbb{Z} / e_{\mathfrak{q}} \mathbb{Z}$;

- $\widehat{H}_{G}^{n}\left(X ; \mathbb{G}_{m}\right)=\prod \mathbb{Z} / e_{\mathfrak{q}} \mathbb{Z}$ pour tout $n$ si $G$ est cyclique. 


\section{B. MORIN}

\subsection{Etude de la suite spectrale de cohomologie équivariante}

On conserve les mêmes notations. La deuxième page de la suite spectrale relative au $G$-faisceau $\mathbb{G}_{m}$ est la suivante :

$$
\begin{array}{cccccc}
\widehat{H}^{-3}(G ; \mathbb{Q} / \mathbb{Z}) & \widehat{H}^{-2}(G ; \mathbb{Q} / \mathbb{Z}) & \widehat{H}^{-1}(G ; \mathbb{Q} / \mathbb{Z}) & \widehat{H}^{0}(G ; \mathbb{Q} / \mathbb{Z}) & \widehat{H}^{1}(G ; \mathbb{Q} / \mathbb{Z}) & \widehat{H}^{-2}(G ; \mathbb{Q} / \mathbb{Z}) \\
0 & 0 & 0 & 0 & 0 & 0 \\
\widehat{H}^{-3}(G ; \mathrm{Cl}(L)) & \widehat{H}^{-2}(G ; \mathrm{Cl}(L)) & \widehat{H}^{-1}(G ; \mathrm{Cl}(L)) & \widehat{H}^{0}(G ; \mathrm{Cl}(L)) & \widehat{H}^{1}(G ; \operatorname{Cl}(L)) & \widehat{H}^{2}(G ; \mathrm{Cl}(L)) \\
\widehat{H}^{-3}\left(G ; U_{L}\right) & \widehat{H}^{-2}\left(G ; U_{L}\right) & \widehat{H}^{-1}\left(G ; U_{L}\right) & \widehat{H}^{0}\left(G ; U_{L}\right) & \widehat{H}^{1}\left(G ; U_{L}\right) & \widehat{H}^{2}\left(G ; U_{L}\right)
\end{array}
$$

On a l'indentification $\widehat{H}^{n}(G ; \mathbb{Q} / \mathbb{Z}) \simeq \widehat{H}^{n+1}(G ; \mathbb{Z})$. Lorsque $G$ est cyclique, la troisième ligne prend les valeurs 0 pour les colonnes d'indice pair et $\widehat{H}^{-2}(G ; \mathbb{Z}) \simeq G^{\text {ab }}=G$ pour celles d'indice impair. On cherche des hypothèses sous lesquelles certaines différentielles sont nulles.

On suppose désormais que le groupe $\mathrm{Cl}(K)$ est trivial. Lorsque $G$ est cyclique, il existe une infinité d'idéaux premiers non nuls inertes de $D$ (c'est une conséquence du théorème de densité de Chebotarev (cf. [Sik03, théorème 2.3(3)])). Cette condition est d'ailleurs nécessaire. On peut alors choisir un point fermé $x$ de $X$ (correspondant à un tel idéal $\mathfrak{p}$ ) fixé par $G$ et de sorte que $\pi: X \rightarrow Y$ soit étale en $x$. Dans ces conditions, $\mathfrak{p}$ est principal et on pose $U=X-\{x\}=\operatorname{Spec}\left(D_{f}\right)$, avec $\mathfrak{p}=D_{f}$. Les groupes de cohomologie étale de $U$ à coefficients dans $\mathbb{G}_{m}$ sont les suivants (cf. [Mil96, II.2.1]) :

$$
H^{q}\left(U ; \mathbb{G}_{m}\right)= \begin{cases}D_{f}^{\times} & \text {pour } q=0, \\ \operatorname{Pic}(U)=\mathrm{Cl}\left(D_{f}\right) & \text { pour } q=1, \\ 0 & \text { pour } q \geqslant 2 .\end{cases}
$$

On désigne toujours par $\widetilde{Z}$ la limite projective des voisinages étales de $Z$ dans $X$. Les $d_{*}^{* *}$ sont les différentielles de la suite spectrale $E_{*}^{* *}(X)$ de cohomologie équivariante de $\mathbb{G}_{m}$ sur $X$. On note aussi $E_{*}^{* *}(\widetilde{Z})$ (respectivement $E_{*}^{* *}(U)$ ) la suite spectrale associée à $\mathbb{G}_{m}$ sur $\widetilde{Z}$ (respectivement sur $U$ ).

Lemme 4.3. Si le groupe $\mathrm{Cl}(K)$ est trivial, la différentielle $d_{2}^{-1 ; 1}$ est nulle. Si de plus le groupe $G$ est cyclique, les différentielles $d_{2}^{n ; 1}$ sont nulles lorsque $n$ est impair.

Démonstration. On considère le morphisme de suites spectrales

$$
h: E_{*}^{* *}(X) \longrightarrow E_{*}^{* *}(\widetilde{Z}) .
$$

On a en particulier le diagramme commutatif suivant.

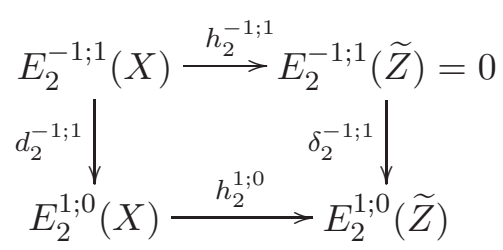

Il suit que $h_{2}^{1 ; 0} \circ d_{2}^{-1 ; 1}=0$. D'autre part, la flèche $h_{2}^{1 ; 0}$ est donnée par le morphisme canonique $\widehat{H}^{1}\left(G ; U_{L}\right) \rightarrow \prod_{\mathfrak{p} \in Z} \widehat{H}^{1}\left(G ; U_{\mathfrak{p}}\right)$, qui est injectif car $\mathrm{Cl}(K)=0$ (cf. lemme 4.22). La deuxième affirmation suit en utilisant la périodicité de la cohomolgie des groupes cycliques.

Lemme 4.4. Si le groupe $\mathrm{Cl}(K)$ est trivial et si $G$ est cyclique, toutes les différentielles $d_{3}^{n ; 3}$ sont nulles. 
UtilisATION D'Une COHOMOLOGIE ÉTALE ÉQUIVARIANTE EN TOPOLOGIE ARITHMÉTIQUE

Démonstration. Sous ces conditions, on dispose du morphisme de suites spectrales

$$
j: E_{*}^{* *}(X) \longrightarrow E_{*}^{* *}(U) .
$$

Comme $\mathfrak{q}$ est principal, le morphisme $\mathrm{Cl}(D) \rightarrow \mathrm{Cl}\left(D_{f}\right)$ est bijectif et induit un isomorphisme $j_{2}^{n+3 ; 1}$ : $\widehat{H}^{n+3}\left(C_{p} ; \mathrm{Cl}(D)\right) \rightarrow \widehat{H}^{n+3}\left(C_{p} ; \mathrm{Cl}\left(D_{f}\right)\right)$. Ce dernier induit à son tour (par restriction aux noyaux des différentielles $d_{2}^{n+3 ; 1}$ et $\delta_{2}^{n+3 ; 1}$ ) le morphisme $j_{3}^{n+3 ; 1}$, qui est donc injectif. Par ailleurs, le même argument que dans la preuve précédente montre que $j_{3}^{n+3 ; 1} \circ d_{3}^{n ; 3}$ est nul, ce qui permet de conclure.

Lemme 4.5. Si le groupe $\mathrm{Cl}(K)$ est trivial, la différentielle $d_{4}^{-3 ; 3}$ est nulle. Si de plus $G$ est cyclique, toutes les différentielles $d_{4}^{n ; 3}$ sont nulles.

Démonstration. Il suffit de montrer que le morphisme $h_{4}^{1 ; 0}$ est injectif.

On vérifie grâce au le lemme 4.3 que les groupes d'arrivée et de départ du morphisme $h_{2}^{1 ; 0}$ : $E_{2}^{1 ; 0}(X) \rightarrow E_{2}^{1 ; 0}(\widetilde{Z})$ restent inchangés jusqu'à la quatrième page. Le morphisme $h_{4}^{1 ; 0}$ s'identifie alors à $h_{2}^{1 ; 0}$, qui est injectif (cf. le lemme 4.22), ce qui montre la première affirmation.

On montre la deuxième en utilisant à nouveau la périodicité de la cohomologie des groupes cycliques et l'égalité $\widehat{H}^{n}(G ; \mathbb{Q} / \mathbb{Z})=0$ lorsque $\mathrm{n}$ est pair.

\subsection{Applications}

Dans tout ce qui suit, $L / K$ est une extension galoisienne de corps de nombres totalement imaginaires de groupe $G$. On note $X$ (respectivement $Y$ ) le spectre de l'anneau d'entiers de $L$ (respectivement de $K$ ).

4.3.1 Majoration et minoration du nombre s d'idéaux premiers ramifiés. On suppose que l'extension $L / K$ est cyclique d'ordre premier $p$. On note $C_{p}$ son groupe de Galois et $s$ le nombre d'idéaux premiers (non nuls) ramifiés dans cette extension.

THÉORŁ̀mE 4.6. On la majoration $s \leqslant 1+\operatorname{dim}_{\mathbb{F}_{p}} \widehat{H}^{1}\left(C_{p} ; U_{L} / \mu\right)+\operatorname{dim}_{\mathbb{F}_{p}} \widehat{H}^{0}\left(C_{p} ; \mathrm{Cl}(L)\right)$.

Démonstration. Le théorème de localisation fournit les isomorphismes (cf. le corollaire 4.2)

$$
\widehat{H}_{G}^{n}\left(X ; \mathbb{G}_{m}\right) \simeq \widehat{H}_{G}^{n}\left(\widetilde{Z} ; \mathbb{G}_{m}\right) \simeq \mathbb{F}_{p}^{s}
$$

La suite spectrale converge vers $\mathbb{F}_{p}^{s}$ en tout degré, donc les groupes $\prod_{i+j=1} E_{\infty}^{i ; j}(X)$ et $\mathbb{F}_{p}^{s}$ ont le même cardinal. Ainsi, l'inégalité (immédiate) $\sharp\left(\prod_{i+j=1} E_{\infty}^{i ; j}(X)\right) \leqslant \sharp\left(\prod_{i+j=1} E_{2}^{i ; j}(X)\right)$ donne la majoration

$$
s \leqslant \operatorname{dim}_{\mathbb{F}_{p}} \widehat{H}^{1}\left(C_{p} ; U_{L}\right)+\operatorname{dim}_{\mathbb{F}_{p}} \widehat{H}^{0}\left(C_{p} ; \mathrm{Cl}(L)\right) .
$$

Le groupe $\mu$ est cyclique, donc $\operatorname{dim}_{\mathbb{F}_{p}} \widehat{H}^{1}\left(C_{p} ; \mu\right) \leqslant 1$. La suite exacte de $C_{p^{-}}$-modules

$$
0 \rightarrow \mu \rightarrow U_{L} \rightarrow U_{L} / \mu \rightarrow 0
$$

donne la suite exacte $\widehat{H}^{1}\left(C_{p} ; \mu\right) \rightarrow \widehat{H}^{1}\left(C_{p} ; U_{L}\right) \rightarrow \widehat{H}^{1}\left(C_{p} ; U_{L} / \mu\right)$ et l'inégalité

$$
\operatorname{dim}_{\mathbb{F}_{p}} \widehat{H}^{1}\left(C_{p} ; U_{L}\right) \leqslant 1+\operatorname{dim}_{\mathbb{F}_{p}} \widehat{H}^{1}\left(C_{p} ; U_{L} / \mu\right)
$$

Les relations (10) et (11) permettent de conclure.

L'étude de la suite spectrale faite dans la $\S 4.2$ est nécessaire pour minorer le nombre $s$.

ThÉORÈme 4.7. Si le groupe $\mathrm{Cl}(K)$ est trivial, alors $s \geqslant 1+\operatorname{dim}_{\mathbb{F}_{p}} \widehat{H}^{0}\left(C_{p} ; \mathrm{Cl}(L)\right)$.

Démonstration. Les lemmes 4.3, 4.4 et 4.5 montrent les égalités suivantes :

$$
E_{\infty}^{-3 ; 3}(X)=E_{2}^{-3 ; 3}(X)=\mathbb{F}_{p} \quad \text { et } \quad E_{\infty}^{-1 ; 1}(X)=E_{2}^{-1 ; 1}(X)=\widehat{H}^{-1}\left(C_{p} ; \mathrm{Cl}(L)\right) .
$$




\section{B. MORIN}

Cette suite spectrale converge vers $\widehat{H}_{C_{p}}^{n}\left(X ; \mathbb{G}_{m}\right) \simeq \mathbb{F}_{p}^{s}$ donc les groupes $\prod_{i+j=0} E_{\infty}^{i ; j}(X)$ et $\mathbb{F}_{p}^{s}$ ont donc le même cardinal. On obtient l'inégalité

$$
s \geqslant 1+\operatorname{dim}_{\mathbb{F}_{p}} \widehat{H}^{1}\left(C_{p} ; \mathrm{Cl}(L)\right) .
$$

De plus, $\widehat{H}^{1}\left(C_{p} ; \mathrm{Cl}(L)\right)$ et $\widehat{H}^{0}\left(C_{p} ; \mathrm{Cl}(L)\right)$ ont le même cardinal car $\mathrm{Cl}(L)$ est fini (voir [Ser68, VIII, proposition 8]).

4.3.2 Lorsque $L$ possède une place finie stable par $G$. Soit alors $U=\operatorname{Spec}\left(D_{f}\right)$ le complémentaire ouvert dans $X$ d'un point fermé $x$ stable sous l'action de $G$. La suite spectrale $\widehat{H}^{p}\left(G ; H^{q}\left(U ; \mathbb{G}_{m}\right)\right) \Longrightarrow \widehat{H}_{G}^{p+q}\left(U ; \mathbb{G}_{m}\right)$ ne possède que deux lignes non nulles. En observant son terme initial, on obtient le résultat suivant.

Proposition 4.8. Si le groupe de Galois (quelquonque) de l'extension $L / K$ fixe une place finie de $L$, on a la suite exacte longue

$$
\begin{aligned}
\cdots \stackrel{d_{2}^{n-2 ; 1} \longrightarrow}{\longrightarrow} \widehat{H}^{n}\left(G ; D_{f}^{\times}\right) \rightarrow \widehat{H}_{G}^{n}\left(U ; \mathbb{G}_{m}\right) \rightarrow \widehat{H}^{n-1}\left(G ; \mathrm{Cl}\left(D_{f}\right)\right) \\
\quad \stackrel{d_{2}^{n-1 ; 1}}{\longrightarrow} \widehat{H}^{n+1}\left(G ; D_{f}^{\times}\right) \rightarrow \widehat{H}_{G}^{n+1}\left(U ; \mathbb{G}_{m}\right) \rightarrow \widehat{H}^{n}\left(G ; \mathrm{Cl}\left(D_{f}\right)\right) \stackrel{d_{2}^{n ; 1}}{\longrightarrow} \cdots
\end{aligned}
$$

Si $L / K$ n'est ramifiée en aucun point de $U$, toutes les différentielles $d_{2}^{n ; 1}$ sont des isomorphismes. Si maintenant $G$ est cyclique, cette suite exacte s'exprime avec le produit des groupes d'inertie pris sur l'ensemble des points fermés de $U / G$. De plus, si $\mathrm{Cl}\left(D_{f}^{G}\right)=0$, le lemme 4.3 s'applique et les différentielles $d_{2}^{n ; 2}$ sont nulles pour $n$ impair. La suite exacte longue se réduit à une suite exacte courte à six termes.

4.3.3 Revêtements cycliques d'une sphère homologique. Dans le contexte de la topologie arithmétique, Ramachandran (cf. [Ram01]) a proposé la définition suivante.

DÉfinition 4.9. Le spectre $Y$ de l'anneau d'entiers d'un corps de nombre $K$ est une 3-sphère $\grave{a}$ homologie entière si $H^{p}\left(Y ; \mathbb{G}_{m}\right)=0$ pour $p \neq 0,3$ et si $H^{0}\left(Y ; \mathbb{G}_{m}\right)$ est de torsion. $Y$ est une 3 -sphère à homologie rationnelle si $H^{0}\left(Y ; \mathbb{G}_{m}\right)$ est de torsion.

D'après [Ram01, Theorem 3], $Y$ est une 3-sphère à homologie entière si et seulement si $K$ est un corps quadratique imaginaire dont le groupe de classes est trivial. Alors $K=\mathbb{Q}(\sqrt{-d})$, où $d$ parcourt l'ensemble $\{1,2,3,7,11,19,43,67,163\}$.

Proposition 4.10. Soient $Y$ une 3-sphère à homologie entière et $L / K$ une extension cyclique de degré $n$. On suppose que $n$ est premier à 2 dans tous les cas et premier à 2 et 3 pour $K=\mathbb{Q}(\sqrt{-3})$. Alors on a les suites exactes

$$
\begin{gathered}
0 \rightarrow \widehat{H}^{1}(G ; \mathrm{Cl}(L)) \rightarrow \prod I_{\mathfrak{q}} \rightarrow G \rightarrow 0, \\
0 \rightarrow \widehat{H}^{1}\left(G ; U_{L}\right) \rightarrow \prod I_{\mathfrak{q}} \rightarrow \mathrm{Cl}(L)^{G} \rightarrow 0,
\end{gathered}
$$

où $\prod I_{\mathfrak{q}}$ désigne le produit des sous-groupes d'inertie dans $G$ indexés sur l'ensemble des places finies de $K$. En particulier, si $n=p$ est premier, on a

$$
\mathrm{Cl}(L)^{C_{p}} \simeq \mathbb{F}_{p}^{s-1} .
$$

Démonstration. Sous ces hypothèses, le groupe $\widehat{H}^{0}\left(G ; U_{L}\right)$ est trivial. En effet, $U_{K}$ est d'ordre 4 pour $d=1$, d'ordre 6 pour $d=3$ et d'ordre 2 sinon. De plus, $\widehat{H}^{0}\left(G ; U_{L}\right)=U_{K} / N\left(U_{L}\right)$ et les éléments de ce groupe sont tués par $n$ qui a été choisi premier au cardinal de $U_{K}$. 
Toutes les différentielles de la suite spectrale $E_{r}^{* *}(X)$ sont nulles pour $r \geqslant 2$. Cette suite spectrale est triviale (i.e. $E_{\infty}^{* *}(X)=E_{2}^{* *}(X)$ ), convergente et il n'y a que deux termes non nuls sur chaque diagonale. On obtient ainsi les deux suites exactes. De plus, pour $n=p$, la première donne

$$
s=1+\operatorname{dim}_{\mathbb{F}_{p}} \widehat{H}^{1}\left(C_{p} ; \mathrm{Cl}(L)\right) .
$$

On vérifie la dernière affirmation grâce aux égalités

$$
\operatorname{dim}_{\mathbb{F}_{p}} \widehat{H}^{1}\left(C_{p} ; \mathrm{Cl}(L)\right)=\operatorname{dim}_{\mathbb{F}_{p}} \widehat{H}^{0}\left(C_{p} ; \mathrm{Cl}(L)\right)=\operatorname{dim}_{\mathbb{F}_{p}} \mathrm{Cl}(L)^{C_{p}},
$$

qui proviennent respectivement de la finitude du groupe de classes $\mathrm{Cl}(L)$ et de l'hypothèse $\mathrm{Cl}(K)=0$.

\subsection{Dualité pour la cohomologie équivariante et preuves analogues}

Nous montrons dans cette section de quelle manière les cohomologies équivariantes ainsi que les suites spectrales relatives aux $G$-faisceaux $\mathbb{Z}$ et $\mathbb{G}_{m}$ respectivement, sont liées par une relation de dualité. Cette dernière provient de dualité d'Artin-Verdier et montre que l'utilisation de ces deux faisceaux revient exactement au même. L'utilisation du $G$-faisceau $\mathbb{Z}$ permet d'obtenir des preuves tout à fait satisfaisantes du point de vue de la topologie arithmétique mais l'utilisation du groupe multiplicatif apparait plus naturelle en cohomologie étale. On conserve les mêmes notations.

4.4.1 Dualité. Ici, le groupe de Galois de l'extension $L / L^{G}$ est un groupe fini quelconque, et $L^{G}$ est totalement imaginaire. On note $M^{D}:=\operatorname{Hom}(M ; \mathbb{Q} / \mathbb{Z})$ le dual d'un groupe abélien de type fini $M$.

Proposition 4.11. On a l'identification $\widehat{H}_{G}^{*}(X ; \mathbb{Z}) \simeq \widehat{H}_{G}^{*}(Z ; \mathbb{Z})$.

Démonstration. Le faisceau $\mathbb{Z}$ est adapté (cf. [Den86, 4.6]). Le résultat est vrai d'après la remarque 3.12 .

LEMme 4.12. On a l'identification $\widehat{H}_{G}^{n}\left(X ; \mathbb{G}_{m}\right) \simeq \widehat{H}_{G}^{n-1}(X ; \mathbb{Z})$.

Démonstration. On note toujours $\Omega$ l'ensemble des premiers de $K$ se ramifiant dans $L$. Pour tout $\mathfrak{q}$ de $\Omega$, on choisit un premier $\mathfrak{p}$ de $L$ au-dessus de $\mathfrak{q}$. Soient $\widetilde{Z}_{\mathfrak{p}}$ la composante connexe de $\widetilde{Z}$ correspondant à $\mathfrak{p}, i: \mathfrak{p} \rightarrow \widetilde{Z}_{\mathfrak{p}}$ l'immersion fermée, $\eta$ l'inclusion du point générique de $\widetilde{Z}_{\mathfrak{p}}$ et $G_{\mathfrak{p}}$ le groupe de décomposition en $\mathfrak{p}$. On a

$$
\widehat{H}_{G}^{n}\left(X ; \mathbb{G}_{m}\right) \simeq \widehat{H}_{G}^{n}\left(\widetilde{Z} ; \mathbb{G}_{m}\right) \simeq \prod_{\Omega} \widehat{H}_{G_{\mathfrak{p}}}^{n}\left(\widetilde{Z}_{\mathfrak{p}} ; \mathbb{G}_{m}\right),
$$

où la deuxième égalité s'obtient en appliquant le lemme de Shapiro sur le terme initial de la suite spectrale $\widehat{H}^{p}\left(G ; H^{q}\left(\widetilde{Z} ; \mathbb{G}_{m}\right)\right) \Longrightarrow \widehat{H}_{G}^{p+q}\left(\widetilde{Z} ; \mathbb{G}_{m}\right)$. On considère maintenant la suite exacte de $G_{\mathfrak{p}}$-faisceaux sur $\widetilde{Z}_{\mathfrak{p}}$

$$
0 \rightarrow \mathbb{G}_{m ; \widetilde{Z}_{\mathfrak{p}}} \rightarrow \eta_{*} \mathbb{G}_{m ; \eta} \rightarrow i_{*} \mathbb{Z} \rightarrow 0 .
$$

Les faisceaux $R^{q}\left(\eta_{*}\right)\left(\mathbb{G}_{m}\right)$ sont nuls pour $q \geqslant 1$ (cf. [Maz73]) et $\eta_{*}$ préserve les $G_{\mathfrak{p}}$-faisceaux injectifs. On obtient donc une $G_{\mathfrak{p}}$-résolution injective de $\eta_{*} \mathbb{G}_{m}$ en appliquant $\eta_{*}$ à une $G_{\mathfrak{p}}$-résolution injective de $\mathbb{G}_{m}$. Ceci permet l'identification $\widehat{H}_{G_{\mathfrak{p}}}^{*}\left(\eta ; \mathbb{G}_{m}\right) \simeq \widehat{H}_{G_{\mathfrak{p}}}^{*}\left(\widetilde{Z}_{\mathfrak{p}} ; \eta_{*} \mathbb{G}_{m}\right)$. Mais $G_{\mathfrak{p}}$ opère sans inertie sur $\eta$ et $\mathbb{G}_{m}$ est adapté sur $\eta$. En effet, $\eta$ est le spectre de $L_{\mathfrak{p}}$, l'hensélisé de $L$ pour la valuation donné par $\mathfrak{p}$ (cf. [End73]). Or le groupe de Galois de ce corps est le même que celui de son complété, qui est de dimension cohomologie stricte égale à 2 . On en déduit

$$
\widehat{H}_{G_{\mathfrak{p}}}^{*}\left(\eta ; \mathbb{G}_{m}\right) \simeq \widehat{H}_{G_{\mathfrak{p}}}^{*}\left(\widetilde{Z}_{\mathfrak{p}} ; \eta_{*} \mathbb{G}_{m}\right)=0
$$

D'autre part, le foncteur $i_{*}$ est exact et préserve les $G_{\mathfrak{p}}$-faisceaux injectifs. On a donc l'identification

$$
\widehat{H}_{G_{\mathfrak{p}}}^{*}(\mathfrak{p} ; \mathbb{Z}) \simeq \widehat{H}_{G_{\mathfrak{p}}}^{*}\left(\widetilde{Z}_{\mathfrak{p}} ; i_{*} \mathbb{Z}\right)
$$




\section{B. MORIN}

La suite exacte longue de cohomologie équivariante associée à (13) ainsi que les relations (14) et (15) donnent les isomorphismes

$$
\widehat{H}_{G_{\mathfrak{p}}}^{n}\left(\widetilde{Z}_{\mathfrak{p}} ; \mathbb{G}_{m ; \widetilde{Z}_{\mathfrak{p}}}\right) \simeq \widehat{H}_{G_{\mathfrak{p}}}^{n-1}(\mathfrak{p} ; \mathbb{Z}) .
$$

De plus, on a

$$
\prod_{\Omega} \widehat{H}_{G_{\mathfrak{p}}}^{n-1}(\mathfrak{p} ; \mathbb{Z}) \simeq \widehat{H}_{G}^{n-1}(Z ; \mathbb{Z}) \simeq \widehat{H}_{G}^{n-1}(X ; \mathbb{Z}),
$$

où la première égalité s'obtient à nouveau en appliquant le lemme de Shapiro sur le terme initial de la suite spectrale (ou directement sur les groupes de cohomologie équivariante (cf. [Bro82, VII, $5]$ ). Finalement, les relations (12), (16) et (17) permettent de conclure.

Remarque 4.13. On a remarqué dans la preuve précédente que $\mathbb{G}_{m}$ était adapté sur $\eta$, où $\eta:=$ $\operatorname{Spec}\left(L_{\mathfrak{p}}\right)$ est le spectre d'un corps local sur lequel un groupe de Galois $G$ opère. Un tel corps est de dimension cohomologique tout au plus deux et son groupe de Brauer s'identifie à $\mathbb{Q} / \mathbb{Z}$ (cf. [Mil96, A.1]). La suite spectrale associée ne possède que deux lignes non nulles. Comme elle converge vers 0 , toutes ses différentielles

$$
d_{3}^{p ; 2}: \widehat{H}^{p}(G ; \mathbb{Q} / \mathbb{Z}) \rightarrow \widehat{H}^{p+3}\left(G ; L_{\mathfrak{p}}^{\times}\right),
$$

sont bijectives. On retrouve les isomorphismes de la théorie du corps de classe local.

LEMme 4.14. On a l'identification $\widehat{H}_{G}^{n}(X ; \mathbb{Z}) \simeq \widehat{H}_{G}^{n-1}(X ; \mathbb{Q} / \mathbb{Z})$.

Démonstration. En considérant la suite spectrale de Leray donnée par l'inclusion du point générique de $X$, on voit que les groupes $H^{q}(X ; \mathbb{Q})$ sont nuls pour $q \geqslant 1$ (cf. [Mil96, II.2.10]). Le terme initial de la suite spectrale est donc nul puisque $\widehat{H}^{*}(G ; \mathbb{Q})=0$. Ainsi, tous les groupes $\widehat{H}_{G}^{q}(X ; \mathbb{Q})$ sont nuls. La suite exacte longue de cohomologie équivariante associée à la suite exacte de $G$-faisceaux

$$
0 \rightarrow \mathbb{Z} \rightarrow \mathbb{Q} \rightarrow \mathbb{Q} / \mathbb{Z} \rightarrow 0
$$

donne le résultat.

ThÉORÈme 4.15. On a l'isomorphisme

$$
\widehat{H}_{G}^{n}\left(X ; \mathbb{G}_{m}\right) \simeq \widehat{H}_{G}^{2-n}(X ; \mathbb{Z})^{D} .
$$

Démonstration. D'après les deux lemmes précédents, il suffit de montrer l'isomorphisme

$$
\widehat{H}_{G}^{n}(X ; \mathbb{Q} / \mathbb{Z}) \simeq \widehat{H}_{G}^{-n}(X ; \mathbb{Z})^{D} .
$$

De la même manière que dans la preuve du lemme 4.12, le théorème de localisation permet de se ramener au spectre d'un corps fini $x=\mathfrak{p}$, sur lequel un groupe de Galois $G$ opère. Les groupes $H^{q}(x ; \mathbb{Q} / \mathbb{Z})$ sont nuls en dimension supérieure à deux et valent $\mathbb{Q} / \mathbb{Z}$ pour $q=0,1$. La suite spectrale

$$
\widehat{H}^{p}\left(G ; H^{q}(x ; \mathbb{Q} / \mathbb{Z})\right) \Longrightarrow \widehat{H}_{G}^{p}(x ; \mathbb{Q} / \mathbb{Z})
$$

ne possède que deux ligne non nulles et se réduit (cf. [CE56, XV, 5.11]) à la suite exacte longue

$$
\cdots \rightarrow \widehat{H}^{n-2}(G ; \mathbb{Q} / \mathbb{Z}) \rightarrow \widehat{H}^{n}(G ; \mathbb{Q} / \mathbb{Z}) \rightarrow \widehat{H}_{G}^{n}(x ; \mathbb{Q} / \mathbb{Z}) \rightarrow \widehat{H}^{n-1}(G ; \mathbb{Q} / \mathbb{Z}) \rightarrow \widehat{H}^{n+1}(G ; \mathbb{Q} / \mathbb{Z}) \rightarrow \cdots
$$

D'après $\left[\right.$ Bro82, VI, 7.3], on a l'identification $\widehat{H}^{n}(G ; \mathbb{Q} / \mathbb{Z}) \simeq \widehat{H}^{-1-n}(G ; \mathbb{Z})^{D}$. En posant $r:=-n$, on obtient

$$
\cdots \rightarrow \widehat{H}^{r+1}(G ; \mathbb{Z})^{D} \rightarrow \widehat{H}^{r-1}(G ; \mathbb{Z})^{D} \rightarrow \widehat{H}_{G}^{-r}(x ; \mathbb{Q} / \mathbb{Z}) \rightarrow \widehat{H}^{r}(G ; \mathbb{Z})^{D} \rightarrow \widehat{H}^{r-2}(G ; \mathbb{Z})^{D} \rightarrow \cdots .
$$

De la même manière, en observant la suite spectrale associé au $G$-faisceau $\mathbb{Z}$ sur $x$, on trouve

$$
\cdots \rightarrow \widehat{H}^{r-2}(G ; \mathbb{Z}) \rightarrow \widehat{H}^{r}(G ; \mathbb{Z}) \rightarrow \widehat{H}_{G}^{r}(x ; \mathbb{Z}) \rightarrow \widehat{H}^{r-1}(G ; \mathbb{Z}) \rightarrow \widehat{H}^{r+1}(G ; \mathbb{Z}) \rightarrow \cdots .
$$


Les deux dernières suites exactes se trouvent être duales l'une de l'autre, ce qui permet d'identifier les groupes $\widehat{H}_{G}^{n}(x ; \mathbb{Q} / \mathbb{Z})$ et $\widehat{H}_{G}^{-n}(x ; \mathbb{Z})^{D}$. On obtient $(18)$ en appliquant le lemme de Shapiro et le théorème de localisation des deux cotés, ce qui achève la preuve du théorème.

D'autre part, les groupes $H^{q}(X ; \mathbb{Z})$ et $H^{3-q}\left(X ; \mathbb{G}_{m}\right)$ sont liés par la dualité d'Artin-Verdier de la manière suivante (cf. [Den86]) :

$$
\begin{gathered}
H^{3}(X ; \mathbb{Z})=H^{0}\left(X ; \mathbb{G}_{m}\right)^{D}=U_{L}^{D}, \quad H^{2}(X ; \mathbb{Z})=H^{1}\left(X ; \mathbb{G}_{m}\right)^{D}=\mathrm{Cl}(L)^{D}, \\
H^{2}\left(X ; \mathbb{G}_{m}\right)=H^{1}(X ; \mathbb{Z})^{D}=0, \quad H^{3}\left(X ; \mathbb{G}_{m}\right)=H^{0}(X ; \mathbb{Z})^{D}=\mathbb{Z}^{D} .
\end{gathered}
$$

De plus, quel que soit le $G$-module $M$, on a $\widehat{H}^{i}\left(G ; M^{D}\right)=\widehat{H}^{-1-i}(G ; M)^{D}$ (cf. [Bro82, VI, 7.3]). On en déduit $E_{2}^{p ; q}\left(X ; \mathbb{G}_{m}\right)=E_{2}^{-1-p ; 3-q}(X ; \mathbb{Z})^{D}$. Vraisemblablement, les différentielles de la première suite spectrale sont données par les applications duales (ou transposées) de la deuxième. On obtient alors la proposition suivante par exactitude du foncteur $\operatorname{Hom}(-; \mathbb{Q} / \mathbb{Z})$.

Proposition 4.16. Quel que soit $2 \leqslant r \leqslant \infty$ et quels que soient les entiers $p$ et $q$, on a

$$
E_{r}^{p ; q}\left(X ; \mathbb{G}_{m}\right)=E_{r}^{-1-p ; 3-q}(X ; \mathbb{Z})^{D} .
$$

4.4.2 Analogues topologiques et preuves analogues. Soit $M$ une variété topologique compacte de dimension trois, fermée, lisse, connexe, orientable et sur laquelle le groupe $C_{p}$ opère fidèlement par automorphismes préservant l'orientation. On note $Z:=M^{C_{p}}$ le lieu de ramification du revêtement $M \rightarrow M / C_{p}$. Il est constitué de $s$ noeuds, dits noeuds ramifiés. Soient de plus $H_{\text {tor }}(M)$ le sous-groupe de torsion de $H_{1}(M ; \mathbb{Z})$ et $H_{\text {free }}(M)$ le quotient $H_{1}(M ; \mathbb{Z}) / H_{\text {tor }}(M)$.

Respectivement, soit $X$ le spectre de l'anneau d'entiers d'un corps de nombre $L$ sur lequel $C_{p}$ opère fidèlement et de sorte que $L^{C_{p}}$ soit totalement imaginaire. On note encore $Z$ le lieu de ramification qui est ici constitué de $s$ places finies ramifiées. Les modules galoisiens $\operatorname{Cl}(L)$ et $U_{L} / \mu$ sont les analogues arithmétiques de $H_{\text {tor }}(M)$ et $H_{\text {free }}(M)$ respectivement. On reprend ci-dessous le théorème 4.6 ainsi que son analogue topologique.

ThÉorème 4.17. En supposant $H_{\text {free }}\left(M / C_{p}\right)=0$ dans le cadre topologique, on a les inégalités suivantes.

$$
\begin{aligned}
& s \leqslant 1+\operatorname{dim}_{\mathbb{F}_{p}} \widehat{H}^{1}\left(C_{p} ; H_{\text {free }}(M)\right)+\operatorname{dim}_{\mathbb{F}_{p}} \widehat{H}^{0}\left(C_{p} ; H_{\text {tor }}(M)\right) \\
& s \leqslant 1+\operatorname{dim}_{\mathbb{F}_{p}} \widehat{H}^{1}\left(C_{p} ; U_{L} / \mu\right)+\operatorname{dim}_{\mathbb{F}_{p}} \widehat{H}^{0}\left(C_{p} ; \mathrm{Cl}(L)\right) .
\end{aligned}
$$

On commence par montrer la première inégalité. On réfère à [Sik03] pour les détails.

Démonstration. Le théorème de localisation (cf. [Swa60, 3.1]) fournit l'isomorphisme

$$
\widehat{H}_{C_{p}}^{n}(M ; \mathbb{Z}) \simeq \widehat{H}_{C_{p}}^{n}(Z ; \mathbb{Z})
$$

Le groupe $C_{p}$ opère trivialement sur $Z$ donc la suite spectrale $\widehat{H}^{i}\left(C_{p} ; H^{j}(Z ; \mathbb{Z})\right) \Longrightarrow \widehat{H}_{C_{p}}^{i+j}(Z ; \mathbb{Z})$ est triviale (cf. [Swa60, 1.1]). Cette suite spectrale ne possède qu'un seul terme non nul sur chaque diagonale, d'ailleurs isomorphe à $\mathbb{F}_{p}^{s}$. On obtient

$$
\widehat{H}_{C_{p}}^{n}(M ; \mathbb{Z}) \simeq \widehat{H}_{C_{p}}^{n}(Z ; \mathbb{Z}) \simeq \mathbb{F}_{p}^{s}
$$

En observant le terme initial de la suite spectrale $\widehat{H}^{i}\left(C_{p} ; H^{j}(M ; \mathbb{Z})\right) \Longrightarrow \widehat{H}_{C_{p}}^{i+j}(M ; \mathbb{Z})$, on obtient immédiatement

$$
s \leqslant 1+\operatorname{dim}_{\mathbb{F}_{p}} \widehat{H}^{0}\left(C_{p} ; H^{2}(M ; \mathbb{Z})\right)+\operatorname{dim}_{\mathbb{F}_{p}} \widehat{H}^{1}\left(C_{p} ; H^{1}(M ; \mathbb{Z})\right) .
$$

La dualité de Poincaré entraine alors (cf. [Sik03, lemme 3.2])

$$
s \leqslant 1+\operatorname{dim}_{\mathbb{F}_{p}} \widehat{H}^{0}\left(C_{p} ; H_{1}(M ; \mathbb{Z})\right)+\operatorname{dim}_{\mathbb{F}_{p}} \widehat{H}^{1}\left(C_{p} ; H_{\text {free }}(M ; \mathbb{Z})\right) .
$$




\section{B. MORIN}

On utilise ensuite l'inégalité

$$
\operatorname{dim}_{\mathbb{F}_{p}} \widehat{H}^{0}\left(C_{p} ; H_{\text {tor }}(M)\right) \leqslant \operatorname{dim}_{\mathbb{F}_{p}} \widehat{H}^{0}\left(C_{p} ; H_{1}(M ; \mathbb{Z})\right)
$$

qui découle de l'hypothèse $H_{\text {free }}\left(M / C_{p}\right)=0$ (cf. [Sik03, preuve du le théorème 1.1(1)]).

La preuve du le théorème 4.6 prend la forme suivante lorsqu'elle est appliquée au faisceau $\mathbb{Z}$.

Démonstration. Le théorème de localisation fournit l' isomorphisme

$$
\widehat{H}_{C_{p}}^{n}(X ; \mathbb{Z}) \simeq \widehat{H}_{C_{p}}^{n}(Z ; \mathbb{Z}) .
$$

Le groupe $C_{p}$ opère trivialement sur $Z$ donc la suite spectrale $\widehat{H}^{i}\left(C_{p} ; H^{j}(Z ; \mathbb{Z})\right) \Longrightarrow \widehat{H}_{C_{p}}^{i+j}(Z ; \mathbb{Z})$ est triviale. Cette suite spectrale ne possède qu'un seul terme non nul sur chaque diagonale, d'ailleurs isomorphe à $\mathbb{F}_{p}^{s}$. On obtient

$$
\widehat{H}_{C_{p}}^{n}(X ; \mathbb{Z}) \simeq \widehat{H}_{C_{p}}^{n}(Z ; \mathbb{Z}) \simeq \mathbb{F}_{p}^{s}
$$

En observant le terme initial de la suite spectrale $\widehat{H}^{i}\left(C_{p} ; H^{j}(X ; \mathbb{Z})\right) \Longrightarrow \widehat{H}_{C_{p}}^{i+j}(X ; \mathbb{Z})$, on obtient immédiatement

$$
s \leqslant \operatorname{dim}_{\mathbb{F}_{p}} \widehat{H}^{1}\left(C_{p} ; H^{2}(X ; \mathbb{Z})\right)+\operatorname{dim}_{\mathbb{F}_{p}} \widehat{H}^{0}\left(C_{p} ; H^{3}(X ; \mathbb{Z})\right)
$$

La dualité d'Artin-Verdier entraine alors

$$
s \leqslant \operatorname{dim}_{\mathbb{F}_{p}} \widehat{H}^{0}\left(C_{p} ; \mathrm{Cl}(L)\right)+\operatorname{dim}_{\mathbb{F}_{p}} \widehat{H}^{1}\left(C_{p} ; U_{L}\right) .
$$

L'inégalité (11)

$$
\operatorname{dim}_{\mathbb{F}_{p}} \widehat{H}^{0}\left(C_{p} ; U_{L}\right) \leqslant 1+\operatorname{dim}_{\mathbb{F}_{p}} \widehat{H}^{0}\left(C_{p} ; U_{L} / \mu\right)
$$

permet de conclure.

On reprend maintenant le théorème 4.7 ainsi que son analogue topologique.

ThÉorème 4.18. Si $H_{1}(M ; \mathbb{Z})$ s'identifie à $H_{\text {free }}(M) \oplus H_{\text {tor }}(M)$ en tant que $C_{p}$-module et si $s \geqslant 1$, alors

$\mathrm{Si} \mathrm{Cl}(K)=0$, alors

$$
s \geqslant 1+\operatorname{dim}_{\mathbb{F}_{p}} \widehat{H}^{0}\left(C_{p} ; H_{\text {tor }}(M)\right) .
$$

$$
s \geqslant 1+\operatorname{dim}_{\mathbb{F}_{p}} \widehat{H}^{0}\left(C_{p} ; \mathrm{Cl}(L)\right) .
$$

De la même manière, la démonstration du théorème 4.7 prend une forme plus agréable lorsqu'elle est appliquée au faisceau $\mathbb{Z}$. Les preuves de ces deux résultats reposent alors sur le théorème de localisation, la dualité de Poincaré (respectivement d'Artin-Verdier) et sur une étude un peu plus fine de la suite spectrale ([Sik03, $\S 3.2]$ et $\S 4.2$ de ce papier) permettant de montrer que certains modules du terme initial survivent à l'infini.

\subsection{Cas des corps de nombres admettant des plongements réels}

Dans cette section, les corps de nombres $L$ et $K=L^{G}$ ne sont plus nécessairement totalement imaginaires. Il est alors indispensable d'utiliser la topologie étale d'Artin-Verdier qui tient compte des places à l'infini. Afin d'alléger l'exposé, nous présentons ici quelques résultats sans démonstrations pour lesquelles nous renvoyons à un travail futur.

Soient $X$ le spectre de l'anneau d'entiers de $L, X_{\infty}$ l'ensemble des places archimédiennes de $L, \bar{X}$ le couple $\left(X ; X_{\infty}\right)$ et $j$ l'inclusion de $X$ dans $\bar{X}$. On munit $\bar{X}$ de la topologie étale d'Artin-Verdier (cf. [Bie87]). Les groupes de cohomologie du groupe multiplicatif $\mathbb{G}_{m ;}:=j_{*} \mathbb{G}_{m}$ sont respectivement $U_{L}, \mathrm{Cl}(L), 0, \mathbb{Q} / \mathbb{Z}$, et 0 en dimension supérieure à trois (cf. [Bie87, 2.7]). Le groupe de Galois de l'extension $L / K$ opère sur $\bar{X}$ et l'on définit la catégorie des $G$-faisceaux pour cette topologie ainsi 
que les groupes de cohomologie équivariante $\widehat{H}_{G}^{*}(\bar{X} ; F)$. Les résultats des $\S \S 2$ et 3 peuvent être appliqués en prenant certaines précautions.

Si $\mathfrak{p}$ est une place finie de $L$, les notations intervenant dans la proposition suivante sont celles qui ont été introduites dans la $\S 4.1$. Si p est une place archimédienne complexe, le couple $(\bar{L} ; \overline{\mathfrak{p}})$ est un hensélisé du corps valué $(L ; \mathfrak{p})$ (cf. [End73]), où $\overline{\mathfrak{p}}$ désigne un prolongement de $\mathfrak{p}$ à $\bar{L}$. On pose alors $U_{\mathfrak{p}}:=\bar{L}^{\times}$et $I_{\mathfrak{p}}^{(a b)}:=I_{\mathfrak{p}}=G_{\mathfrak{p}} \subseteq G_{\overline{\mathfrak{p}}} \simeq \mathbb{Z} / 2 \mathbb{Z}$.

Proposition 4.19. Soit $G$ un groupe fini opérant fidèlement sur $\bar{X}$. On a les isomorphismes ci-dessous, où les deux premiers produits sont pris sur toutes les places de $K$ et le troisième sur l'ensemble des places finies de $K$ :

- $\widehat{H}_{G}^{*}\left(\bar{X} ; \mathbb{G}_{m ; \bar{X}}\right) \simeq \prod \widehat{H}^{*}\left(G_{\mathfrak{p}} ; U_{\mathfrak{p}}\right)$;

- $\widehat{H}_{G}^{0}\left(\bar{X} ; \mathbb{G}_{m ; \bar{X}}\right) \simeq \prod I_{\mathfrak{q}}^{(a b)}$;

- $\widehat{H}_{G}^{1}\left(\bar{X} ; \mathbb{G}_{m ; \bar{X}}\right) \simeq \prod \mathbb{Z} / e_{\mathfrak{q}} \mathbb{Z}$.

Tous les résultats de la $\S 4.3$ se généralisent ainsi à toutes les extensions de corps de nombres avec de légères modifications. Par exemple, si $L / K$ est une extension de corps de nombres de groupe $C_{p}$, on a la proposition suivante, où $s$ (respectivement $\bar{s}$ ) désigne le nombre de places finies (respectivement finies et infinies) ramifiées.

Proposition 4.20. On a la majoration $s \leqslant 1+\operatorname{dim}_{\mathbb{F}_{p}} \widehat{H}^{1}\left(C_{p} ; U_{L} / \mu\right)+\operatorname{dim}_{\mathbb{F}_{p}} \widehat{H}^{0}\left(C_{p} ; \mathrm{Cl}(L)\right)$.

Si le groupe $\mathrm{Cl}(K)$ est trivial, alors $\bar{s} \geqslant 1+\operatorname{dim}_{\mathbb{F}_{p}} \widehat{H}^{0}\left(C_{p} ; \mathrm{Cl}(L)\right)$.

On considère à nouveau l'action (fidèle) du groupe $C_{p}$ sur une 3-variété $M$ et sur un corps de nombres $L$ respectivement, en gardant les notations (et les hypothèses) précédentes. On note aussi $s_{0}$ le nombre de places finies ramifiées dans cette extension.

Proposition 4.21. Si $H_{\text {free }}(M)=0$ et si $s \geqslant 1$, alors

$$
H_{\mathrm{tor}}(M)^{C_{p}} \simeq \mathbb{F}_{p}^{s-1} \text {. }
$$

Si $U_{L} / \mu=0$, alors $p=2, s_{0} \geqslant 1$ et

$$
\mathrm{Cl}(L)^{C_{2}} \simeq \mathbb{F}_{2}^{s_{0}-1}
$$

Démonstration. Dans le cadre topologique, les hypothèses $H_{\text {free }}(M)=0$ et $s \geqslant 1$ assurent que la suite spectrale $E_{2}^{* *}(M ; \mathbb{Z})$ est triviale (cf. [Sik03, 3.3 et 3.4]). De la même manière, l'hypothèse $U_{L} / \mu=0$ permet de montrer que la suite spectrale $E_{2}^{* *}\left(\bar{X} ; \mathbb{G}_{m}\right)$ est triviale. Il faut ici utiliser les résultats de la section 4.2 généralisés aux corps de nombres quelconques et considérer de plus le morphisme de suites spectrales induit par l'inclusion du point générique de $\bar{X}$ (l'ensemble des valuations de $L$ ), pour montrer que toutes les différentielles sont nulles.

Le fait que les deux suites spectrales soient triviales donne immédiatement les deux résultats précédents.

\subsection{Complément technique}

Lemme 4.22. Soient $L / K$ une extension galoisienne de corps de nombres de groupe $G, D$ et $R$ les anneaux d'entiers de $L$ et $K$ respectivement. On suppose que $\mathrm{Cl}(K)$ est trivial. On note $U_{L}$ (respectivement $U_{K}$ ) le groupe des unités de $L$ (respectivement de $K$ ). Soient $\left(\mathfrak{q}_{j}\right)_{1 \leqslant j \leqslant r}$ les idéaux premiers finis de $K$ qui se ramifient dans l'extension $L / K$ et $\left(\mathfrak{p}_{i}\right)_{1 \leqslant i \leqslant s}$ les idéaux premiers de $L$ au-dessus des $\mathfrak{q}_{j}$. Soit $U_{\mathfrak{p}_{i}}$ (respectivement $U_{\mathfrak{q}_{j}}$ ) le groupe des unités de l'hensélisé $D_{\mathfrak{p}_{i}}^{h}$ de l'anneau local $D_{\left(\mathfrak{p}_{i}\right)}$ (respectivement de l'hensélisé $R_{\mathfrak{q}_{j}}^{h}$ de l'anneau local $\left.R_{\left(\mathfrak{q}_{j}\right)}\right)$, $L_{i}$ et $K_{j}$ les corps de fractions de $D_{\mathfrak{p}_{i}}^{h}$ et $R_{\mathfrak{q}_{j}}^{h}$. 


\section{B. MORIN}

Si $\mathrm{Cl}(K)$ est trivial, alors le morphisme canonique $H^{1}\left(G ; U_{L}\right) \rightarrow H^{1}\left(G ; \prod_{1 \leqslant i \leqslant s} U_{\mathfrak{p}_{i}}\right)$ est injectif.

Démonstration. Soit $X^{0}$ l'ensemble des places finies de $L$. Le groupe de Galois opère sur $X^{0}$ et on a le morphisme de $\mathbb{Z}[G]$-modules div : $L^{\times} \rightarrow \sum_{\mathfrak{p} \in X^{0}} \mathbb{Z}$ donné par les valuations. On note $W$ l'image du morphisme div. On a le morphisme de suites exactes de $\mathbb{Z}[G]$-modules suivant.

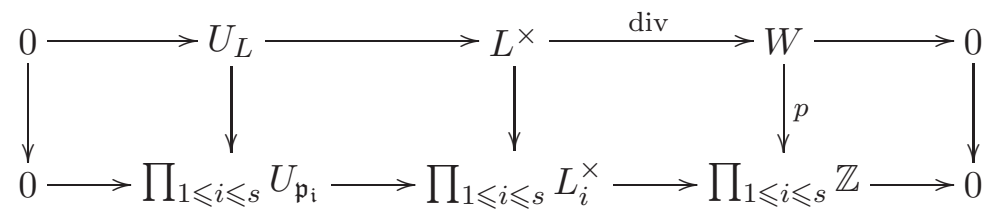

En utilisant le théorème de Hilbert 90 et le fait que les $H^{q}(G ; \ldots)$ forment un foncteur cohomologique, on obtient le morphisme de suites exactes suivant.

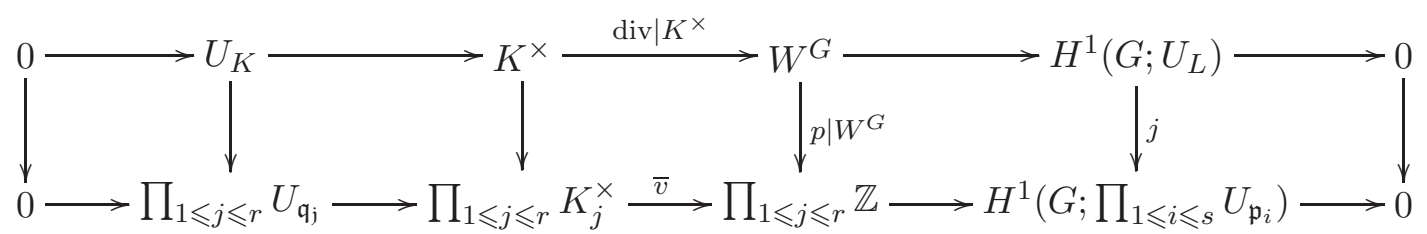

Alors $j$ s'identifie au morphisme

$$
W^{G} / \operatorname{Im}\left(\operatorname{div} \mid K^{\times}\right) \longrightarrow\left(\prod_{1 \leqslant i \leqslant r} \mathbb{Z}\right) / \operatorname{Im}(\bar{v})
$$

induit par $p$, dont on montre facilement qu'il est injectif lorsque $\mathrm{Cl}(K)$ est trivial.

\section{Conclusion}

Nous donnons ici une interprétation des résultats et de leurs preuves exposés dans ce travail, afin d'essayer d'éclaircir et d'approfondir le dictionnaire de la topologie arithmétique.

\subsection{Quelques éléments du dictionnaire}

5.1.1 Dans les versions précédentes du dictionnaire de la topologie arithmétique [Ram01, Rez99], le groupe de Galois $G_{L}^{\mathrm{nr}}$ de l'extension maximale non ramifiée en toutes les places (archimédiennes et ultramétriques) d'un corps de nombre $L$ est vu comme l'analogue du groupe fondamental topologique de la variété 'correspondante' $M$. En effet, il s'agit du groupe fondamental pour la topologie étale d'Artin-Verdier. En suivant cette idée, $H_{1}(M ; \mathbb{Z})=\pi_{1}(M)^{\text {ab }}$ devrait être l'analogue du groupe de Galois de l'extension abélienne maximale non ramifiée de $L$. Par la théorie du corps de classe, ce groupe s'identifie à $\mathrm{Cl}(L)$. Il n'y aurait donc pas de place pour le groupe des unités. Cette même analogie conduit à montrer que la version arithmétique de la conjecture de Poincaré est fausse (cf. [Ram01]).

C'est peut-être pour éviter des contradictions de ce type que A. Reznikov a proposé dans la deuxième version du dictionnaire [Rez00,12], de voir un corps de nombre $L$ comme une 3-variété $M$ bordant une 4-variété $N$ de sorte que le morphisme $\pi_{1}(M) \rightarrow \pi_{1}(N)$ soit surjectif, pour considérer les invariants cohomologiques de $N$ (et non de $M$ ). Cependant, le non-sens évoqué ci-dessus est toujours présent dans ce deuxième dictionnaire.

Comme nous le précisons ci-dessous, le travail exposé dans ce papier confirme clairement la première version [Rez99] du dictionnaire, et écarte la deuxième [Rez00]. En effet, toutes les preuves sont basées sur la cohomologie de $\bar{X}$ et de $M$ pour aboutir à des résultats respectant parfaitement le dictionnaire [Rez99]. 
5.1.2 Nous soutenons les analogies suivantes.

Les corps de nombres, les extensions galoisiennes de corps de nombres $L / L^{G}$, les places finies, les places finies ramifiée et l'ensemble des places finies ramifiés doivent être vus respectivement comme des 3-variétés, des revêtements ramifiés galoisiens de 3 -variétés $M \rightarrow M / G$, des noeuds dans $M$, des noeuds ramifiés et comme le lieu de ramification (plus précisément, les sous-groupes de décomposition et d'inertie se correspondent). Ici, le terme 3-variété doit probablement prendre un sens plus large.

De plus, $\mathrm{Cl}(L)$ et $U_{L} / \mu$ correspondent, de manière compatible à l'action d'un groupe de Galois, aux groupes $H_{\text {tor }}(M)$ et $H_{\text {free }}(M)$. D'ailleurs, le paragraphe suivant confirme à nouveau ces deux dernières correspondances.

\subsection{Cohomologie de Lichtenbaum et topologie arithmétique}

5.2.1 Considérons la topologie Weil-étale. Les résultats de Lichtenbaum dans [Lic05] montrent que les groupes de cohomologie à support compact de $\bar{X}$ à coefficients entiers devraient être les suivants (cf. [Lic05, 6.3]).

$$
H_{c}^{q}(\bar{X} ; \mathbb{Z})= \begin{cases}0 & \text { pour } q=0, \\ \left(\prod_{X_{\infty}} \mathbb{Z}\right) / \mathbb{Z} & \text { pour } q=1, \\ \operatorname{Pic}^{1}(\bar{X})^{\mathcal{D}} & \text { pour } q=2, \\ \left(\mu_{L}\right)^{\mathcal{D}} & \text { pour } q=3, \\ 0 & \text { pour } q \geqslant 4 .\end{cases}
$$

Ci-dessus, $\operatorname{Pic}^{1}(\bar{X})$ est le noyau du morphisme du groupe de classe d'Arakelov dans $\mathbb{R}^{\times}$donné par la valeur absolue. On note aussi $A^{\mathcal{D}}:=\operatorname{Hom}(A ; \mathbb{R} / \mathbb{Z})$ le dual de Pontryagin d'un groupe topologique abélien séparé et localement compact $A$. D'autre part, le sous-groupe de torsion et le quotient libre de $H_{c}^{2}(\bar{X} ; \mathbb{Z})$ sont donnés par la suite exacte (cf. [Lic05, 6.4])

$$
0 \rightarrow \mathrm{Cl}(L)^{\mathcal{D}} \rightarrow \operatorname{Pic}^{1}(\bar{X})^{\mathcal{D}} \rightarrow \operatorname{Hom}\left(U_{L} ; \mathbb{Z}\right) \rightarrow 0 .
$$

D'après l'interprétation de Deninger dans [Den06, 7], la cohomologie de Lichtenbaum correspond à la cohomologie des faisceaux sur $M$, qui s'identifie d'ailleurs à la cohomologie singulière, car une variété topologique est un espace localement contractile. Puisque $M$ est une variété compacte, la cohomologie à support compact s'identifie à la cohomologie usuelle. Ainsi, $H^{2}(M ; \mathbb{Z})$ doit être l'analogue du groupe $\operatorname{Pic}^{1}(\bar{X})^{\mathcal{D}}$. Lorsque $M$ est orientable, la dualité de Poincaré identifie $H^{2}(M ; \mathbb{Z})$ et $H_{1}(M ; \mathbb{Z})$ en tant que groupes abéliens. Dans la situation équivariante, l'action du groupe de Galois sur ces deux groupes se trouve être inversée à travers cet isomorphisme. De plus, $H_{c}^{1}(\bar{X} ; \mathbb{Z})$ s'identifie à $\operatorname{Hom}\left(U_{L} / \mu ; \mathbb{Z}\right)$, c'est-à-dire l'analogue de $\operatorname{Hom}\left(H_{\text {free }}(M ; \mathbb{Z}) ; \mathbb{Z}\right)=\operatorname{Hom}\left(\pi_{1}(M) ; \mathbb{Z}\right)$.

5.2.2 D'autres éléments du dictionnaire. D'après le paragraphe précédent, les analogues arithmétiques des groupes $H^{1}(M ; \mathbb{Z})$ et $H^{2}(M ; \mathbb{Z})$ devraient être $\operatorname{Hom}\left(U_{L} / \mu ; \mathbb{Z}\right)$ et $\operatorname{Pic}^{1}(\bar{X})^{\mathcal{D}}$ respectivement. Si l'on voit $\bar{X}$ comme un espace orientable, le groupe $H_{1}(M ; \mathbb{Z})$ devrait être l'analogue de $\operatorname{Pic}^{1}(\bar{X})^{\mathcal{D}}$. L'action d'un éventuel groupe de Galois $G$ sur ce dernier devrait se faire à travers le groupe opposé à $G$, afin d'être compatible à l'action de $G$ sur $H_{1}(M ; \mathbb{Z})$. De plus, $\mathrm{Cl}(L)$ et $U_{L}$ correspondent respectivement aux groupes $H_{\text {tor }}(M)$ et $H_{\text {free }}(M)$ déduits de $H_{1}(M ; \mathbb{Z})$. Une sphère à homologie entière (respectivement rationnelle) est un corps de nombres dont l'analogue de $H_{1}(M ; \mathbb{Z})$ est nul (respectivement de torsion).

Enfin, le groupe $G_{L}^{\text {nr }}$ ne serait pas l'analogue de $\pi_{1}(M)$ (puisque les abélianisés de ces groupes ne se correspondent pas), mais nous espérons pouvoir revenir plus tard sur cette question. 


\section{B. MORIN}

Remarque 5.1. Soit $\bar{X} \rightarrow \bar{Y}$ un morphisme au-dessus de $\overline{\operatorname{Spec}(\mathbb{Z})}$ donné par une extension de corps de nombre $L / K / \mathbb{Q}$. L'inclusion $I_{K} \hookrightarrow I_{L}$ induit morphisme $\operatorname{Pic}^{1}(\bar{X})^{\mathcal{D}} \rightarrow \operatorname{Pic}^{1}(\bar{Y})^{\mathcal{D}}$. Ainsi, ce groupe dépend fonctoriellement de $\bar{X}$ et de manière covariante.

\subsection{Comparaison des hypothèses}

Les résultats de Sikora étudiés dans ce travail donnent une majoration (4.17), une minoration (4.18) et une égalité dans un cas particulier (4.21), du nombre $s$ de places (respectivement de noeuds) ramifiées dans un revêtement cyclique d'ordre premier. Pour la majoration et l'égalité, les hypothèses faites en topologie sont strictement plus fortes qu'en arithmétique. De plus, d'après [Sik03, 5], elles sont toutes nécessaires en topologie. Malheureusement, nous n'avons pas été en mesure de démontrer (à l'aide des mêmes méthodes) la minoration de $s$ en arithmétique à partir de l'analogue des hypothèses topologiques (qui peuvent être formulées grâce au paragraphe précédent).

Néanmoins, ce travail donne le sentiment que le cadre topologique est 'plus général' que celui des corps de nombres. En effet, l'arithmétique apparaît ici beaucoup plus rigide que le cadre topologique. Cette idée vague est d'ailleurs nettement confirmée dans [Den06]. On peut aussi illustrer ce fait par les observations suivantes. Un corps de nombres est de manière unique un revêtement de $\mathbb{Q}$, alors qu'une 3-variété orientable peut s'obtenir d'un grand nombre de manières différentes comme revêtement de $\mathbb{S}^{3}$. Il existe exactement dix sphères à homologie entière en arithmétique (toutes de degré un ou deux) et une infinité en topologie (cf. [Ram01]). L'analogue de $H_{1}(M ; \mathbb{Z})$ est plus gros que le groupe de Galois de l'extension abélienne maximale non ramifiée de $L$.

Il est d'ailleurs amusant d'imaginer à quoi ressembleraient les preuves des mêmes résultats basées sur la topologie Weil-étale. En supposant que la ligne $E_{2}^{i ; 3}(\bar{X} ; \varphi ! \mathbb{Z})=\widehat{H}^{i}\left(C_{p} ; \mu_{L}\right)$ soit non nulle et qu'elle survive à l'infini, on obtiendrait exactement les mêmes démonstrations dans les deux contextes.

On se rend alors compte que les différences entre les preuves arithmétiques et topologiques que nous avons proposées proviennent des 'défauts' de la cohomologie étale. Cependant, lorsqu'il n'y pas de ramification à l'infini, ces mêmes défauts n'apparaissent pas dans les groupes de cohomologie étale équivariante modifiée. En effet, ces derniers donnent les 'bons' groupes liés à la ramification (c'est à dire les mêmes que dans le cadre topologique), ce qui renforce l'analogie des preuves que nous avons proposées. On vérifie cette affirmation en utilisant le théorème de localisation et en observant que les termes initiaux des suites spectrales (par exemple à coefficients dans $\mathbb{Z}$ ), définies sur le spectre d'un corps fini et sur un cercle munis d'une action d'un groupe fini $G$, sont en fait les mêmes. Cette analogie n'est d'ailleurs pas respectée par les (quatre premiers) groupes de cohomologie étale équivariante non modifiée. En effet, si $G$ opère trivialement, on a $H^{1}\left(\operatorname{Spec}\left(\mathbb{F}_{q}\right)_{\text {et }} ; G ; \mathbb{Z}\right)=0$ et $H^{1}\left(\mathbb{S}^{1} ; G ; \mathbb{Z}\right)=\mathbb{Z}$, comme le montre la suite spectrale [Gro57, 5.2.9].

Lorsqu'il y a de la ramification à l'infini, les places complexes apparaissent comme des points dans la cohomologie équivariante modifiée.

\subsection{Places finies et places archimédiennes}

5.4.1 Dans le topos associé à la topologie Weil-étale sur $\bar{X}$, une place finie est donnée par l'inclusion fermée du topos classifiant $B_{\mathbb{Z}}$. Par ailleurs, l'espace classifiant du groupe discret $\mathbb{Z}$ est le cercle $\mathbb{S}^{1}$, dont le topos est homotopiquement équivalent à $B_{\mathbb{Z}}$ (cf. [Moe95, IV.1.1]). Il est donc naturel de voir géométriquement une place finie comme l'immersion fermée d'un cercle.

5.4.2 D'après Ramachandran, on sait que $\bar{X}$ doit être vu comme la compactification d'une variété non compacte correspondant à $X$. 
Dans le topos associé à la topologie étale (d'Artin-Verdier) sur $\bar{X}$, une place archimédienne est donnée par l'inclusion du topos ponctuel, c'est-à-dire un point du topos étale (cf. [GAV72, IV.6]). C'est la raison pour laquelle les calculs de cohomologie étale font apparaître les places archimédiennes comme des points, les bouts d'une 3-variété non compacte.

Cette analogie offre quelques contradictions. D'une part, toute variété orientable de dimension trois se réalise comme revêtement ramifié de $\mathbb{S}^{3}$ dont le lieu de ramification est composé de noeuds. Or un corps de nombres non totalement réel est, de manière unique, un revêtement de $\mathbb{Q}$ ramifié à l'infini. Par exemple, une extension quadratique imaginaire de $\mathbb{Q}$ ramifiée en au moins un premier ne peut pas être vue comme une variété orientable $M$ au-dessus de $M / C_{2}=\mathbb{S}^{3}$. En effet, dans cette situation, le lieu de ramification ne peut pas être constitué de noeuds et d'un point (isolé dans le lieu de ramification). D'autre part, les places archimédiennes doivent être comptées comme composantes du lieu de ramification dans la proposition 4.20, et doivent être ignorées dans la proposition 4.21.

Il semble donc que le fait de voir un corps de nombres comme une simple variété orientable pose quelques problèmes dans l'interprétation des places archimédiennes. D'ailleurs, dans les travaux de Deninger (cf. [Den02] et [Den06]), il n'est pas clair qu'un corps de nombres corresponde à une variété proprement dite.

De plus, dans le topos associé à la topologie Weil-étale, une place archimédienne devrait être donnée par l'inclusion du topos classifiant $B_{\mathbb{R}}$ du groupe topologique $\mathbb{R}$, qui est loin d'être équivalent au topos ponctuel.

5.4.3 Toujours dans les travaux de Deninger, une place archimédienne correspond à un point sur lequel $\mathbb{R}$ opère trivialement, ce qui donne lieu au topos $B_{\mathbb{R}}$ (cf. [GAV72, IV.2.5]). Respectivement, une place finie $\mathfrak{p}$ correspond à l'immersion fermée d'un cercle $\mathbb{R} / \log (N \mathfrak{p}) \mathbb{Z}=\mathbb{R} / l(\mathfrak{p}) \mathbb{Z}$ sur lequel $\mathbb{R}$ opère naturellement. Le topos associé est le topos induit sur l'objet $\mathbb{R} / l(\mathfrak{p}) \mathbb{Z}$ de $B_{\mathbb{R}}$ (cf. [GAV72, IV.5.1]). Ce dernier topos est canoniquement équivalent à $B_{l(\mathfrak{p}) \mathbb{Z}}(\mathrm{cf}$. [GAV72, IV.5.8]). Par ailleurs, dans le topos associé à la topologie Weil-étale, une place finie $\mathfrak{p}$ est donnée plus exactement par l'immersion fermée de $B_{W_{k(\mathfrak{p})}}$. De plus, en normalisant convenablement, le logarithme de la valeur absolue donne $W_{k(\mathfrak{p})}=\log (N \mathfrak{p}) \mathbb{Z}=l(\mathfrak{p}) \mathbb{Z} \subseteq \mathbb{R}$. Ces deux points de vue sont donc compatibles.

\section{REMERCIEMENTS}

Je tiens à remercier Boas Erez pour m'avoir initié à la topologie arithmétique et pour m'avoir proposé cette problématique. Je suis également très reconnaissant envers Lorenzo Ramero. Ses lectures attentives et ses nombreuses remarques ont été très utiles tout au long de ce travail. Je tiens aussi à remercier Matthias Flach, pour m'avoir transmis ses résultats non publiés sur la cohomologie du groupe de Weil.

\section{REFERENCES}

Bie87 M. Bienenfeld, An étale cohomology duality theorem for number fields with a real embedding, Trans. Amer. Math. Soc. 303 (1987), 71-96.

Bro82 K. S. Brown, Cohomology of groups, Graduate Texts in Mathematics (Springer, London, 1982).

CE56 H. Cartan and S. Eilenberg, Homological algebra (Princeton University Press, Princeton, NJ, 1956).

Den86 C. Deninger, An extension of Artin-Verdier duality to nontorsion sheaves, J. reine angew. Math. 366 (1986), 18-31.

Den02 C. Deninger, A note on arithmetic topology and dynamical systems, in Algebraic number theory and algebraic geometry, Contemporary Mathematics, vol. 300 (American Mathematical Society, Providence, RI, 2002), 99-114. 
Den06 C. Deninger, A dynamical systems analogue of Lichtenbaum's conjectures on special values of Hasse-Weil zeta functions, Preprint (2006), arXiv:math/0605724.

End73 O. Endler, On Henselization of valued fields, Bol. Soc. Brasil. Mat. 4 (1973), 97-109.

Fla06 M. Flach, Cohomology of topological groups with applications to the Weil group, Preprint (2006), Compositio Math., to appear.

God58 R. Godement, Théorie des faisceaux (Hermann, Paris, 1958).

Gro57 A. Grothendieck, Sur quelques points d'algèbre homologique, Tôhoku Math. J. (2) 9 (1957), 119-221.

Gro66 A. Grothendieck, Classes de Chern et représentations linéaires des groupes discrets, in Dix exposés sur la cohomologie des schémas (North-Holland, Amsterdam/Masson, Paris, 1966), 215-306.

Gro71 A. Grothendieck, Revêtements étales et groupe fondamenal (SGA1), Lectures Notes in Mathematics, vol. 224 (Springer, New York, 1971).

GAV72 A. Grothendieck, M. Artin and J. L. Verdier, Théorie des Topos et cohomologie étale des schémas (SGA4), Lectures Notes in Mathematics, vols 269, 270, 305 (Springer, New York, 1972).

Hub93 R. Huber, Étale cohomology of Henselian rings and cohomology of abstract Riemann surfaces of fields, Math. Ann. 295 (1993), 703-708.

Lic05 S. Lichtenbaum, The Weil-etale topology for number rings, Preprint (2005), arXiv:math/0503604.

Maz73 B. Mazur, Notes on étale cohomology of number fields, Ann. Sci. École Norm. Sup. (4) 6 (1973), $521-556$.

Mil80 J. S. Milne, Étale cohomology. Princeton Mathematical Series, No. 33 (Princeton University Press, Princeton, NJ, 1980).

Mil96 J. S. Milne, Arithmetic duality theorems, Perspectives in Mathematics, vol. 1 (Academic Press, Boston, MA, 1996).

Moe95 I. Moerdijk, Classifying spaces and classifying topoi, Lecture Notes in Mathematics, vol. 1616 (Springer, New York, 1995).

Ram01 N. Ramachandran, A note on arithmetic topology, C. R. Math. Acad. Sci. Soc. R. Can. 23 (2001), 130-135.

Ray70 M. Raynaud, Anneaux locaux henséliens, Lecture Notes in Mathematics, vol. 169 (Springer, Heidelberg, 1970).

Rez99 A. Reznikov, Hakenness and $b_{1}$, Preprint (1999), arXiv:math/9903064.

Rez00 A. Reznikov, Embedded incompressible surfaces and homology of ramified covering of 3-manifolds, Selecta Math. (N.S.) 6 (2000), 1-39.

Ser65 J. P. Serre, Cohomologie Galoisienne, Lectures Notes in Mathematics, vol. 11 (Springer, New York, 1965).

Ser68 J. P. Serre, Corps locaux (Hermann, Paris, 1968).

Sik03 A. Sikora, Analogies between group action on 3-manifolds and number fields, Comment. Math. Helv. 78 (2003), 832-844.

Str84 R. Strano, On the étale cohomology of Hensel rings, Comm. Algebra 12 (1984), 2195-2211.

Swa60 R. Swan, A new method in fixed point theory, Comment. Math. Helv. 34 (1960), 1-16.

Wei95 C. A. Weibel, An introduction to homological algebra, Cambridge Studies in Advanced Mathematics, vol. 38 (Cambridge University Press, Cambridge, 1995).

Baptiste Morin Baptiste.Morin@math.u-bordeaux1.fr

Institut de Mathématiques de Bordeaux, UMR 5251, Université Bordeaux 1, 351 Cours de la Libération, 33405 Talence Cedex, France 